\title{
CMB anisotropy experiments
}

\author{
George F. Smoot \\ Lawrence Berkeley National Lab \& Physics Department, University of California, Berkeley CA 94720, USA
}

\begin{abstract}
Anisotropies in the cosmic microwave background (CMB) encode information about the evolution and development of the universe. Quality observations of CMB anisotropies can provide a very strong test of cosmological models and provide high precision determination of major cosmological parameters. This paper provides a review of the COBE DMR results, the current status of the measurements of the CMB anisotropy power spectrum and then focuses current and future programs including both suborbital observations and the two selected satellite missions: the NASA MidEX mission MAP and the ESA M3 mission Max Planck Surveyor. This review includes both a description of the experimental programs and the expected quality level of results. (C) 2000 Elsevier Science B.V. All rights reserved.
\end{abstract}

PACS: $98.80 .-\mathrm{v}$

Keywords: Cosmic microwave background radiation; Cosmology; Schramm

\section{Introduction}

The observed cosmic microwave background (CMB) radiation provides strong evidence for the big bang model of cosmology and is the best probe we have for determining conditions in the early universe as well as determining many important cosmological parameters. The angular power spectrum of the CMB contains information on virtually all cosmological parameters of interest, including the geometry of the universe $(\Omega)$, the baryon density $\left(\Omega_{\mathrm{b}}\right)$, the Hubble expansion rate $(h)$, the cosmological constant $(\Lambda)$, the number of light neutrinos $\left(n_{v}\right)$, the ionization history of the universe, and the amplitudes and spectral indices of the primordial and tensor perturbation spectra. Precise CMB observations, data analysis, and interpretation can distinguish between cosmological models. They can be used to verify that the range of models under consideration is plausible and to distinguish between models with primordial perturbations (e.g. the inflationary

E-mail address: smoot@astro.1bl.gov (G.F. Smoot). 
big bang) or those with active perturbations (e.g. topological defects which must result from spontaneous symmetry breaking of unified forces). Once a model is thus singled out, its parameters can, in principle, be determined to accuracies of the order of a per cent [41].

Since the initial detection of CMB temperature anisotropies by the COBE DMR [75], over a dozen other balloon-borne and ground-based experiments have reported anisotropy detections on smaller angular scales. With the existence of anisotropies now firmly established, observational goals have shifted towards an accurate determination of the CMB anisotropy power spectrum over a wide range of angular scales. The reasons for this are two-fold: (1) If the processes producing the initial fluctuations are stochastic and random phase, then the power spectrum contains all the information of the underlying physical model. (2) It is observationally easier to obtain a power spectrum than a fully reliable map. Several technical advances, including improved receivers, interferometry, and long-duration balloon flights, hold great promise for high-precision maps in the next few years. Ultimately, the two approved satellites: NASA MidEX mission MAP and ESA M3 mission Planck are expected to provide high-angular-resolution high-sensitivity maps of the entire sky in multifrequency bands. Thus, we can anticipate increasingly complex data sets requiring sophisticated analysis: COBE DMR 4-year maps (6144 pixels), the CfPA balloon experiments MAXIMA/BOOMERANG (26,000 to 130,000 pixels), MAP $(\approx 400,000$ pixels), and Planck ( $>10^{6}$ pixels). These maps then hold the promise of revolutionizing cosmology in terms of making it significantly more precise in quantitative terms.

This paper is intended to provide the background necessary to understand the existing data, data soon to data from experiments in progress, and finally the data forthcoming from the more advanced experiments and space missions. We proceed with some historical context, a review of the COBE observations both for the discovery of anisotropy and as a prototype for the next generation of space missions, a review of the current and proposed generations of balloon-borne experiments and interferometers, and finally a discussion of the new space-based experiments. General literature is presented in Refs. [1-90], CMB anisotropy experiment references can be found in [91-122]. Additional information is available at http://aether.lbl.gov/www/.

\section{CMB background}

Primordial nucleosynthesis calculations require a cosmic background radiation (CBR) with a temperature $k T \sim 1 \mathrm{MeV}$ at a redshift of $z \sim 10^{9}$. Gamow et al. [3] realized that this CBR was required and predicted its evolution to a faint residual relic radiation with a current temperature of a few degrees. Our more modern view of the hot big bang models gives the cosmic background radiation a very central role in the development of the universe.

The CMB was serendipitously discovered by Penzias and Wilson [65] in 1964 (published in $1965)$ and they noted that it was isotropic to the sensitivity of their measurement $(<10 \%)$. The observed CMB spectrum is well characterized by a $2.73 \pm 0.01 \mathrm{~K}$ blackbody spectrum. The hot big bang model predicts that the CBR should have a thermal spectrum and this is verified precisely. Combined with the observed spectrum of the dipole anisotropy, this precision thermal spectrum also provides us with the knowledge and ability to separate CMB anisotropies from the various foregrounds. Anisotropies will in general have a spectrum set by the derivative of the CMB frequency spectrum. 


\section{Theoretical anisotropies}

There are three primary threads of science that are pursued by CMB anisotropy measurements:

(1) Initial conditions for large-scale structure formation: The formation of galaxies, clusters of galaxies, and large scale structures is a key issue in cosmology. Theory indicates that whatever the seeds of structure formation, they will leave their imprint as anisotropies in the CMB. Different scenarios for structure formation will leave different anisotropies.

(2) Physics of the early universe: $\mathrm{CMB}$ anisotropy measurements are a probe of the ultra-high energy physics and processes that occur in the very early universe. These observations are a probe of inflation or quantum gravity and a test of potential topological defects (monopoles, strings, domain walls, and textures) that must result from spontaneous symmetry breaking.

(3) Geometry and dynamics of the universe: Observations of CMB anisotropies provide information on the metric and topology of the space-time, the isotropy of expansion of space-time, the curvature of space, and the possible rotation and shear of the universe.

There is now a fairly extensive literature on the first two areas and the third is what we consider classical cosmology. In the early history of the field all measurements led only to upper limits on $\mathrm{CMB}$ anisotropy and these in turn led to limits on but not a measurement of these processes.

A major finding of the initial COBE DMR discovery [75] was that the CMB was anisotropic on all observed angular scales. A key question is what these anisotropies represent. Immediately, the interpretation focused upon the seeds of large-scale structure formation. In the early 1970s the observed large-scale structure and scaling arguments led to the prediction [63,34,90] that the primordial gravitational potential perturbations must have an equal rms amplitude on all scales. This corresponds to a matter density perturbation power-law spectrum, $P(k) \propto k^{n}$, where $k$ is the comoving wave number, with $n=1$. At that time there were no known mechanisms for producing such a scale-invariant power spectrum of fluctuations. In 1982 it was found that inflationary models predicted nearly scale-invariant perturbations as a result of quantum mechanical fluctuations at very early times. Even with the proliferation of inflationary models, it is found that essentially all reasonable inflationary models predict $n \approx 1$. Presumably, a more reasonable class of inflationary models will result in requiring a tie to particle physics. It is now known that topological defects naturally produce scale-invariant fluctuations. Thus, there are at least two known mechanisms for producing a nearly scale-invariant primordial perturbation spectrum.

The translation from a scale-invariant spectrum of perturbations to the CMB temperature anisotropies depends upon angular scale and the contents of the universe. On large angular scales the results of most models are fairly similar. Including the effects of a standard cold dark matter model. This is consistent with the power spectrum measured by the COBE DMR and other published CMB data. The observed power spectrum of fluctuation amplitudes is also consistent with models of large-scale structure formation based upon primordial seeds produced by quantum fluctuations or topological defects in the early universe.

The physics of anisotropy caused by primordial density perturbations is usually divided into four generic areas although they are all treated properly in the full Sachs-Wolfe effect [70]. These effects are: the gravitational redshift which dominates at large angular scales, the (Rees-Sciama) effect on light propagating through a changing potential, the Doppler effect caused by the motion of the observer or the source, and temperature or entropy variations. 
Models of the formation of structure in the universe fall into two broad classes: inflationary and defect models. Each model predicts an angular power spectrum of CMB anisotropy described in terms of the amplitude of the spherical harmonic of multipole order $\ell$.

The most detailed theoretical work has been carried out for inflationary models. Hu et al. [40] have argued that all inflationary models produce an angular power spectrum with a unique set of "doppler" or "acoustic" peaks between $\ell=100$ and $1000\left(11^{\prime}<\theta<1.8^{\circ}\right)$. The relative position and height and the detailed shape of the peaks provides more independent constraints than there are parameters in the inflationary scenario and allow their determination.

Defect models, including cosmic strings and textures, provide an alternative to inflation. Such models predict a non-Gaussian distribution of temperature fluctuations and a power spectrum different from that of inflation.

Most cosmological models do not predict the exact CMB temperature pattern that would be observed in our sky, but rather predict a statistical distribution of anisotropies. In the context of such models, the CMB temperature observed in our sky is only a single realization drawn from the cosmic statistical distribution. Theoretical models most often predict a power spectrum in spherical harmonic amplitudes; as the physics of the models leads to primordial fluctuations that are Gaussian random fields, the power spectrum is sufficient to characterize the results. Observations of the sky can be expressed as a spherical harmonic temperature expansion $T(\theta, \phi)=$ $\sum_{\ell m} a_{\ell m} Y_{\ell m}(\theta, \phi)$. If the original perturbations are Gaussian random fields, the $a_{\ell m}$ are Gaussianly distributed, and the power at each $\ell$ is $(2 \ell+1) C_{\ell} /(4 \pi)$, where $C_{\ell} \equiv\left\langle\left|a_{\ell m}\right|^{2}\right\rangle$, is sufficient to characterize the results. For an idealized full-sky observation, the variance of each measured $C_{\ell}$ is $[2 /(2 \ell+1)] C_{\ell}^{2}$. This sampling variance (known as cosmic variance) comes about because each $C_{\ell}$ is chi-squared distributed with $(2 \ell+1)$ degrees of freedom for our observable volume of the universe [85]. Thus, in addition to experimental uncertainties, we account for the cosmic sample variance uncertainties due to our observation of a single realization in our analyses of the DMR maps. Cosmic variance exists independently of the quality of the experiment. The power spectrum from the 4-year DMR map is cosmic variance limited for $\ell \lesssim 20$. For $\ell \gtrsim 20$ no observations approach the cosmic variance limit yet.

\section{The legacy of COBE}

The cosmic background explorer (COBE) was NASA's first satellite dedicated to cosmology and is a milestone for that and for the legacy of information that it has provided on the early universe. Much of its results are from observations of the cosmic microwave background (CMB). The CMB is a pillar of the big bang model and encodes information about energy release in the early universe, primordial perturbations, and the geometry of the universe. COBE's legacy of the precise measurement of the CMB spectrum and the discovery and early mapping of the CMB anisotropy low- $\ell$ power spectrum provides a position from which to carry out a program testing our cosmological theories and understanding the early universe precisely.

The differential microwave radiometers (DMR) experiment [74] discovered CMB anisotropies from analysis of its first year of data $[75,9,87,44]$. The CMB temperature fluctuations were measured at an angular resolution of $7^{\circ}$ at frequencies of $31.5,53$, and $90 \mathrm{GHz}$. These results were supported by a detailed examination of the DMR calibration and its uncertainties [10] and 
a detailed treatment of the upper limits on residual systematic errors [44]. The COBE results were confirmed by the positive cross-correlation between the COBE data and data from balloon-borne observations at a shorter wavelength [32] and later by comparison of the COBE data and data from the ground-based Tenerife experiment [52] at longer wavelengths. The positive correlation at both longer and shorter wavelengths provides confidence in the results as being true CMB anisotropy and not a confusing foreground such as Galactic emission. The results from analysis of two years of DMR data [7] reconfirmed the results from the first year data.

\subsection{The COBE DMR instruments and data analysis}

The DMR consists of 6 differential microwave radiometers: 2 nearly independent channels, labeled $\mathrm{A}$ and $\mathrm{B}$, at frequencies $31.5,53$, and $90 \mathrm{GHz}$ (wavelength $9.5,5.7$, and $3.3 \mathrm{~mm}$, respectively). Each radiometer measures the difference in power between two $7^{\circ}$ fields of view separated by $60^{\circ}$, $30^{\circ}$ to either side of the spacecraft spin axis [74]. Fig. 1 shows a schematic signal path for the DMRs. COBE was launched from Vandenberg Air Force Base on 18 November 1989 into a $900 \mathrm{~km}, 99^{\circ}$ inclination circular orbit, which precesses to follow the terminator (light dark line on the Earth) as the Earth orbits the Sun. Attitude control keeps the spacecraft pointed away from the Earth and nearly perpendicular to the sun with a slight backward tilt so that solar radiation never directly illuminates the aperture plane. The combined motions of the spacecraft spin ( $75 \mathrm{~s}$ period), orbit ( $103 \mathrm{~m}$ period), and orbital precession ( $\sim 1^{\circ}$ per day) allow each sky position to be compared to all others through a highly redundant set of temperature difference measurements spaced $60^{\circ}$ apart. The on-board processor box-car integrates the differential signal from each channel for $0.5 \mathrm{~s}$, and records the digitized differences for daily playback to a ground station.

Ground data processing consists of calibration, extensive systematic error analyses, and conversion of time-ordered data to sky maps [46]. Checks on the correlated noise in the maps [51] due to the map-making process indicate they are well below the $1 \%$ level. The DMR time-ordered data include systematic effects such as emission from the Earth and Moon, the instrument's response to thermal changes, and the instrument's response to the Earth's magnetic field. The largest detected effects do not contribute significantly to the DMR maps; they are either on time scales long compared to the spacecraft spin sampling (e.g. thermal gain drifts) or have time dependence inconsistent with emission fixed on the celestial sphere (e.g. magnetic effects). Detected and potential systematic effects were quantitatively analyzed in detail [46]. Data with the worst systematic contamination (lunar emission, terrestrial emission, and thermal gain changes) were not used in the map-making process and constitute less than $10 \%$ of the data in the 53 and $90 \mathrm{GHz}$ channels. The remaining data were corrected using models of each effect. The data editing and correction parameters were conservatively chosen so that systematic artifacts, after correction, are less than $6 \mu \mathrm{K} \mathrm{rms}(95 \%$ confidence upper limit) in the final DMR map in the worst channel. This is significantly less than the levels of the noise and celestial signals.

A dipole $T_{\mathrm{d}}=3.356 \mathrm{mK}$ anisotropy signal (thermodynamic temperature in Galactic coordinates Cartesian components $[X, Y, Z]=[-0.2173,-2.2451,+2.4853] \mathrm{mK})$ is subtracted from the time-ordered differential data prior to forming the 4-year sky maps to reduce spatial gradients within a single pixel. A small residual dipole remains in the maps from a combination of CMB and Galactic emission. The mean signal-to-noise ratios in the $10^{\circ}$ smoothed maps are approximately 


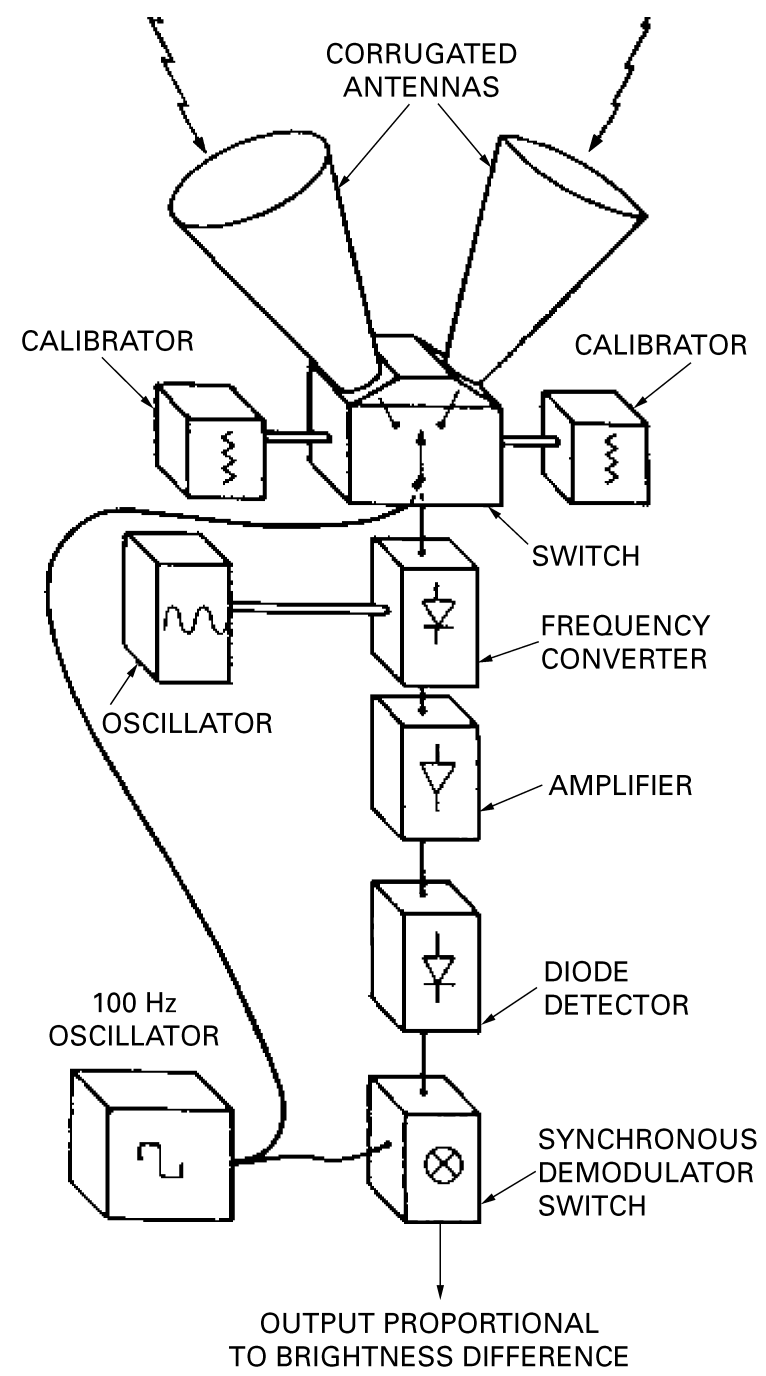

Fig. 1. DMR signal flow schematic.

$0.5,1.5$, and 1.0 for 31,53 , and $90 \mathrm{GHz}$, respectively. For a multi-frequency co-added map the signal-to-noise ratio is $\sim 2$. This signal-to-noise level is adequate to portray an accurate overall visual impression of the anisotropy. Visual comparison of the full sky maps at each frequency, after averaging the $\mathrm{A}$ and $\mathrm{B}$ channels, removing the $\mathrm{CMB}$ dipole, and smoothing to $10^{\circ}$ effective resolution show coincident features. Well off the Galactic plane these are clearly true CMB anisotropy features. Simulated data in combination with the noise appropriate to 1-, 2-, and 4-years of DMR $53 \mathrm{GHz}$ observations show the convergence of the DMR maps with the input simulated data. Increasing years of data result in the emergence of the input large-scale features. We can be confident that the large-scale features in the 4-year DMR maps are real features rather than confusing noise. 
Kogut et al. [47] examine the Galactic contamination of the high Galactic latitude regions of the DMR maps which remain after the Galactic emission cut is applied. No significant crosscorrelation is found between the DMR maps and either the $408 \mathrm{MHz}$ synchrotron map.

A significant correlation is found between the DMR maps and the dust-dominated DIRBE $140 \mu \mathrm{m}$ map, with frequency dependence consistent with a superposition of dust and free-free emission. The correlation is really with a component of Galactic emission with a spectral index of about -2.1 which could be very flat spectrum synchrotron as expected where cosmic-ray electron acceleration is actually occurring. We use the term free-free to stand for this component which corresponds to a $7^{\circ} \mathrm{rms}$ free-free emission component of $7.1 \pm 1.7 \mu \mathrm{K}$ at $53 \mathrm{GHz}$ and a dust component of $2.7 \pm 1.3 \mu \mathrm{K}$ at $53 \mathrm{GHz}$. Since this emission is uncorrelated with CMB anisotropies it constitutes $<10 \%$ of the CMB power. The amplitude of the correlated free-free component at $53 \mathrm{GHz}$ agrees with a noisier estimate of free-free emission derived from a linear combination of DMR data which includes all emission with free-free spectral dependence. The combined dust and free-free emission contribute $10 \pm 4 \mu \mathrm{K} \mathrm{rms}$ at both 53 and $90 \mathrm{GHz}$, well below the $30 \mu \mathrm{K}$ cosmic signal. These Galactic signal analyses are consistent with the fact that the fitted cosmological parameters are nearly unaffected by removal of modeled Galactic signals $[33,36]$ with the notable exception of the quadrupole, which has significant Galactic contamination [47]. A search by Banday et al. [4] finds no evidence for significant extragalactic contamination of the DMR maps.

\subsection{Summary of 4-year COBE DMR CMB measurements}

(1) The full 4-year set of COBE DMR observations is analyzed and full sky maps have been produced [6]. The typical signal-to-noise ratio in a $10^{\circ}$ smoothed frequency-averaged map is $\sim 2$, enough to provide a good visual impression of the anisotropy.

(2) The DMR (despite its being a differential instrument) finds a CMB monopole temperature of $T_{0}=2.725 \pm 0.020 \mathrm{~K}$ [46]. This is in excellent agreement with the COBE FIRAS precision measurement of the spectrum of the CMB, $T_{0}=2.728 \pm 0.002 \mathrm{~K}$ [31].

(3) The CMB dipole from DMR has amplitude $3.358 \pm 0.024 \mathrm{mK}$ toward Galactic coordinates $(l, b)=\left(264^{\circ} .31 \pm 0^{\circ} .17,48^{\circ} .05 \pm 0^{\circ} .10\right), \quad$ or equatorial coordinates $(\alpha, \delta)=\left(11^{\mathrm{h}} 11^{\mathrm{m}} 257^{\mathrm{s}} \pm 23 \mathrm{~s}\right.$, $-7^{\circ} .22 \pm 0^{\circ} .08$ ) epoch $\mathrm{J} 2000$. This is consistent with the dipole amplitude and direction derived by COBE FIRAS [31].

(4) The $95 \%$ confidence interval for the observed $\ell=2$ quadrupole amplitude is $4 \mu \mathrm{K} \leq Q_{\mathrm{rms}} \leq 28 \mu \mathrm{K}$. This is consistent with the value predicted by a power-law fit to the power spectrum yields a quadrupole normalization of: $Q_{\mathrm{rms}-\mathrm{PS}}=15.3_{-2.8}^{+3.8} \mu \mathrm{K}[47,36]$.

(5) The power spectrum of large angular scale CMB measurements is consistent with an $n=1$ power-law $[33,36,88]$. If the effects of a standard cold dark matter model are included, COBE DMR should find $n_{\text {eff }} \approx 1.1$ for a $n=1$ universe. With full use of the multi-frequency 4-year DMR data, including our estimate of the effects of Galactic emission, we find a power-law spectral index of $n=1.2 \pm 0.3$ and a quadrupole normalization $Q_{\text {rms-Ps }}=15.3_{-2.8}^{+3.8} \mu \mathrm{K}$. For $n=1$ the best-fit normalization is $\left.Q_{\text {rms-Ps }}\right|_{n=1}=18 \pm 1.6 \mu \mathrm{K}$. Differences in the derived values of $Q$ and $n$ between various analyses of DMR data are much more dependent on the detailed data selection effects than on the analysis technique.

(6) The DMR data are consistent with Gaussian statistics. Gaussian statistics are favored over other toy statistical models by a factor of $\sim 5[48]$. 


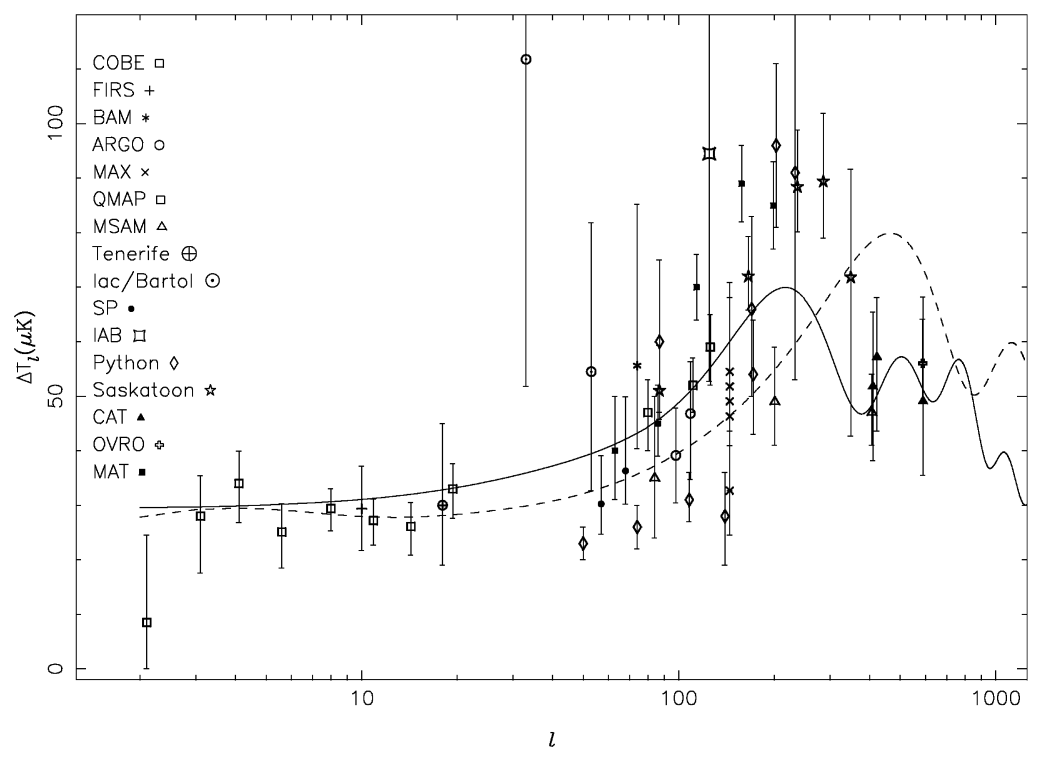

Fig. 2. Current status of CMB anisotropy observations: plotted are the band power amplitudes of CMB anisotropy detections. The error bars correspond to the $68 \%$ confidence level. The solid curve indicates the expected spectrum for a standard flat CDM model $\left(\Omega_{0}=1, \Omega_{\mathrm{b}}=0.05, h=0.5\right)$ and the dashed curve is an open $(\Omega=0.3)$ CDM model. This gives a strong indication that the geometery of the universe is closer to flat than well open. A true comparison of data with models should involve convolution of these curve with each experimental filter function.

\subsection{COBE conclusions}

The COBE-discovered [75] higher-order $(\ell \geq 2)$ anisotropy is interpreted as being the result of perturbations in the energy density of the early universe, manifesting themselves at the epoch of the CMB's last scattering. These perturbations are the seeds of large-scale structure formation and are relics from processes occurring in the very early universe at extremely high energies. In the standard scenario the last scattering of cosmic background photons takes place at a redshift of approximately 1100 , at which epoch the large number of photons was no longer able to keep the hydrogen sufficiently ionized. The optical thickness of the cosmic photosphere is roughly $\Delta z \sim 100$ or about 10 arcminutes, so that features smaller than this size are damped. Observations of the CMB anisotropy power spectrum can reveal to us much of the interesting history of the early universe and so a great deal of effort has gone into its observation.

\section{Current anisotropy power spectrum}

More than 10 experiments have now observed CMB anisotropies. Anisotropies are observed on angular scales larger than the minimum 10' damping scale (see Fig. 2) and are consistent with those expected from an initially scale-invariant power spectrum of potential and thus metric fluctuations. It is believed that the large-scale structure in the universe developed through the process of gravitational instability where small primordial perturbations in energy density were amplified by 
gravity over the course of time. The initial spectrum of density perturbations can evolve significantly in the epoch $z>1100$ for causally connected regions (angles $\lesssim 1^{\circ} \Omega_{\text {tot }}^{1 / 2}$ ). The primary mode of evolution is through adiabatic (acoustic) oscillations, leading to a series of peaks that encode information about the perturbations and geometry of the universe, as well as information on $\Omega_{0}, \Omega_{\mathrm{b}}, \Omega_{A}$ (cosmological constant), and $H_{0}$ [71]. The location of the first acoustic peak is predicted to be at $\ell \sim 220 \Omega_{\mathrm{tot}}^{-1 / 2}$ or $\theta \sim 1^{\circ} \Omega_{\mathrm{tot}}^{1 / 2}$ and its amplitude increases with increasing $\Omega_{\mathrm{b}}$.

Only somewhat weak conclusions can be drawn based on the current smaller angular scale data (see Fig. 2). However, new data are being acquired at an increasing rate. With future experiments and the prospect of next generation satellite missions: MAP and Planck, a precise measurement of the CMB anisotropy power spectrum is possible and likely, allowing us to decode the information that it contains $[43,41]$.

\section{Current and near-term experiments}

Many groups have been and are working to measure the anisotropy. Though some are focussed on large angular scales at frequencies not observed with DMR, most concentrate on smaller angular scales. Table 1 contains a list for recent, current and planned experiments modified from that compiled by Page [61].

Rather than review all the experiments, we focus here on a representative sample.

\subsection{MAX/MAXIMA/BOOMERANG}

The MAX/MAXIMA/BOOMERANG payloads are representative of current and currently planned balloon-borne missions and are somewhat further along.

\subsubsection{MAX}

The Millimeter-wave Anisotropy eXperiment (MAX) is a balloon-borne bolometric instrument which observes at multiple frequencies with high sensitivity on the $0.5^{\circ}$ angular scale. MAX has completed five flights detecting significant CMB fluctuations [30,2,58,24,18,78,50].

The MAX instrument consists of an off-axis Gregorian telescope and a bolometric photometer mounted on an altitude-controlled balloon-borne platform which makes measurements at an altitude of $36 \mathrm{~km}$. The Gregorian telescope consists of a $1-\mathrm{m}$ primary and a nutating elliptical secondary. The underfilled optics provide a $0.55^{\circ} \mathrm{FWHM}$ beam when focused and aligned. The $5.7 \mathrm{~Hz}$ nutation of the secondary modulates the beam on the sky sinusoidally though $\pm 0.68^{\circ}$ and the altitude control sweeps the beam over a $6^{\circ}$ or $8^{\circ}$ path and back in about $108 \mathrm{~s}$, producing about 15-20 independent temperature differences on the sky. Depending upon the time of observation and location of the region under observation sky rotation can cause the observed region to be in the shape of a bow-tie.

On flights 4 and 5 the single-pixel four-band bolometric receiver featured negligible sensitivity to radio frequency interference and an adiabatic demagnetization refrigerator to cool the photometer to $85 \mathrm{mK}$. The dichroic photometer used for MAX has $(\delta v / v)$ of $0.57,0.45,0.35$, and 0.25 filter bands at $3.5,6,9$, and $15 \mathrm{~cm}^{-1}$, respectively. MAX covers the high-frequency side of the window formed 
Table 1

Recently completed, current and planned anisotropy experiments ${ }^{\mathrm{a}}$

\begin{tabular}{|c|c|c|c|c|c|}
\hline Experiment & Resolution & Frequency & Detectors & Type & Groups \\
\hline $\operatorname{ACBAR}(p)[91]$ & $4^{\prime}$ & $150 \mathrm{GHz}$ & Bol & $\mathrm{C} / \mathrm{B}$ & UCB, UCSB, Caltech \\
\hline $\mathrm{ACE}(\mathrm{c})[92]$ & $0.2^{\circ}$ & $25-100 \mathrm{GHz}$ & HEMT & $\mathrm{C} / \mathrm{B}$ & UCSB \\
\hline Archeops(p) [94] & $0.33^{\circ}$ & $30 \mathrm{GHz}$ & HEMT & $\mathrm{I} / \mathrm{G}$ & Chicago \\
\hline APACHE(c) [93] & $0.33^{\circ}$ & $90-400 \mathrm{GHz}$ & Bol & $\mathrm{C} / \mathrm{G}$ & $\begin{array}{l}\text { Bologna, Bartol } \\
\text { Rome III }\end{array}$ \\
\hline $\operatorname{ARGO}(\mathrm{f})[95]$ & $0.9^{\circ}$ & $140-3000 \mathrm{GHz}$ & Bol & $\mathrm{C} / \mathrm{B}$ & Rome I \\
\hline $\operatorname{ATCA}(f)[96]$ & $0.03^{\circ}$ & $8.7 \mathrm{GHz}$ & HEMT & $\mathrm{I} / \mathrm{G}$ & CSIRO \\
\hline $\mathrm{BAM}(\mathrm{c})[97]$ & $0.75^{\circ}$ & $90-300 \mathrm{GHz}$ & Bol & $\mathrm{C} / \mathrm{B}$ & $\mathrm{UBC}, \mathrm{CfA}$ \\
\hline $\operatorname{Bartol}(\mathrm{c})$ [109] & $2.4^{\circ}$ & $90-270 \mathrm{GHz}$ & Bol & $\mathrm{C} / \mathrm{G}$ & Bartol \\
\hline $\operatorname{BEAST}(\mathrm{p})[92]$ & $0.2^{\circ}$ & $25-100 \mathrm{GHz}$ & HEMT & $\mathrm{C} / \mathrm{B}$ & UCSB \\
\hline BOOMERanG(c) [100] & $0.2^{\circ}$ & $90-400 \mathrm{GHz}$ & Bol & $\mathrm{C} / \mathrm{G}$ & $\begin{array}{l}\text { Rome I, Caltech, } \\
\text { UCB, UCSB }\end{array}$ \\
\hline $\operatorname{CAT}(\mathrm{c})[101]$ & $0.17^{\circ}$ & $15 \mathrm{GHz}$ & HEMT & $\mathrm{I} / \mathrm{G}$ & Cambridge \\
\hline $\mathrm{CBI}(\mathrm{p})[102]$ & $0.0833^{\circ}$ & $26-36 \mathrm{GHz}$ & HEMT & $\mathrm{I} / \mathrm{G}$ & Caltech, Penn. \\
\hline $\mathrm{CG}(\mathrm{c})[103]$ & $1^{\prime} \times 0.8^{\prime}$ & $0.6-22 \mathrm{GHz}$ & HEMT & $\mathrm{C} / \mathrm{G}$ & Ratan 600 \\
\hline DASI(p) [105] & $0.33^{\circ}$ & $30 \mathrm{GHz}$ & HEMT & $\mathrm{I} / \mathrm{G}$ & Chicago \\
\hline FIRS(f) $[106]$ & $3.8^{\circ}$ & $170-680 \mathrm{GHz}$ & Bol & $\mathrm{C} / \mathrm{B}$ & $\begin{array}{l}\text { Chicago, MIT, Princeton, } \\
\text { NASA/GSFC }\end{array}$ \\
\hline HACME/SP(f) [107] & $0.6^{\circ}$ & $30 \mathrm{GHz}$ & HEMT & $\mathrm{C} / \mathrm{G}$ & UCSB \\
\hline $\mathrm{IAB}(\mathrm{f})[108]$ & $0.83^{\circ}$ & $150 \mathrm{GHz}$ & Bol & $\mathrm{C} / \mathrm{G}$ & Bartol \\
\hline $\operatorname{MAT}(\mathrm{p})[110]$ & $0.2^{\circ}$ & $30-150 \mathrm{GHz}$ & HEMT/SIS & $\mathrm{C} / \mathrm{G}$ & Penn, Princeton \\
\hline $\operatorname{MAX}(\mathrm{f})[111]$ & $0.5^{\circ}$ & $90-420 \mathrm{GHz}$ & Bol & $\mathrm{C} / \mathrm{B}$ & UCB, UCSB \\
\hline MAXIMA(c) [112] & $0.2^{\circ}$ & $90-420 \mathrm{GHz}$ & Bol & $\mathrm{C} / \mathrm{B}$ & $\begin{array}{l}\text { UCB, Rome I } \\
\text { Caltech, UCSB }\end{array}$ \\
\hline $\operatorname{MSAM}(\mathrm{c})$ [113] & $0.4^{\circ}$ & $40-680 \mathrm{GHz}$ & Bol & $\mathrm{C} / \mathrm{B}$ & $\begin{array}{l}\text { Chicago, Bartol, } \\
\text { Brown, Princeton, } \\
\text { NASA/GSFC }\end{array}$ \\
\hline OVRO 40/5(c) [114] & $0.033^{\circ}, 0.12^{\circ}$ & $15-35 \mathrm{GHz}$ & HEMT & $\mathrm{C} / \mathrm{G}$ & Caltech, Penn \\
\hline PYTHON(c) [118] & $0.75^{\circ}$ & $35-90 \mathrm{GHz}$ & Bol/HEMT & $\mathrm{C} / \mathrm{G}$ & $\begin{array}{l}\text { Carnegie Mellon } \\
\text { Chicago, UCSB }\end{array}$ \\
\hline QMAP(f) [119] & $0.2^{\circ}$ & $20-150 \mathrm{GHz}$ & HEMT/SIS & $\mathrm{C} / \mathrm{B}$ & Princeton, Penn \\
\hline SASK(f) $[121]$ & $0.5^{\circ}$ & $20-45 \mathrm{GHz}$ & HEMT & $\mathrm{C} / \mathrm{G}$ & Princeton \\
\hline SuZIE(c) [123] & $0.017^{\circ}$ & $150-300 \mathrm{GHz}$ & Bol & $\mathrm{C} / \mathrm{G}$ & Caltech \\
\hline TopHat(p) [125] & $0.33^{\circ}$ & $150-700 \mathrm{GHz}$ & Bol & $\mathrm{C} / \mathrm{B}$ & $\begin{array}{l}\text { Bartol, Brown, } \\
\text { DSRI, Chicago, } \\
\text { NASA/GSFC }\end{array}$ \\
\hline Tenerife(c) [124] & $6.0^{\circ}$ & $10-33 \mathrm{GHz}$ & HEMT & $\mathrm{C} / \mathrm{G}$ & NRAL, Cambridge \\
\hline Viper(c) $[126]$ & $0.33^{\circ}$ & $30 \mathrm{GHz}$ & HEMT & $\mathrm{I} / \mathrm{G}$ & Chicago \\
\hline $\operatorname{VLA}(\mathrm{c})[127]$ & $0.0028^{\circ}$ & $8.4 \mathrm{GHz}$ & HEMT & $\mathrm{I} / \mathrm{G}$ & Haverford, NRAO \\
\hline $\operatorname{VSA}(p)[128]$ & - & $30 \mathrm{GHz}$ & HEMT & $\mathrm{I} / \mathrm{G}$ & Cambridge \\
\hline White Dish(f) [129] & $0.2^{\circ}$ & $90 \mathrm{GHz}$ & Bol & $\mathrm{C} / \mathrm{G}$ & Carnegie Mellon \\
\hline
\end{tabular}

a(i) For "Type" the first letter distinguishes between configuration or interferometer, the second between ground or balloon. (ii) An "f" after the experiment's name means it's finished; a "c" denotes current; a "p" denotes planned, building may be in progress but there is no data yet. 
by Galactic dust emission rising at higher frequencies and Galactic synchrotron and free-free emission increasing at lower frequencies. The $15 \mathrm{~cm}^{-1}$ channel acts as a guard against Galactic dust and atmospheric emission. The multiple frequencies have sufficient redundancy to provide confidence that the signal is CMB and not a foreground or systematic effect.

MAX is calibrated both by an on-board commandable membrane and by observations of planets, usually Jupiter. The two techniques agree at roughly the $10 \%$ level. The calibration is such that the quoted temperature difference is the real temperature difference on the sky.

MAX makes deep CMB observations (typically one hour) on regions generally selected to be low in dust contrast and total emission and free from known radio sources. MAX has made observations on five flights. The data from most of the scans are in good agreement but the scan of the $\mu$-Pegasi region is significantly lower than the rest. A combination of all the data seems to be coming out at an intermediate value between GUM and $\mu$-Pegasi regions and may all be consistent with coming from a single parent population [80].

The center of the scan is the same for the three observations of GUM (the star Gamma Ursae Minoris) but the relative geometry is such that the three scans made bow-tie patterns which cross at the star. White and Bunn [84] have made use of this fact to construct a two-dimensional map of the region which is roughly $10^{\circ} \times 5^{\circ}$. The title of their paper is "A First Map of the CMB at $0.5^{\circ}$ Resolution". Since then a map covering 180 square degrees was generated by Tegmark et al. [83] using the Saskatoon data.

Making maps is clearly the appropriate approach for the current generation of new experiments. MAX has evolved to new systems MAXIMA and BOOMERANG, which are designed and constructed for the goal of getting the power spectrum around the first "Doppler" peak and beyond and making maps covering a significant portion of the sky.

\subsubsection{MAXIMA}

MAXIMA stands for MAX imaging system. The current one-dimensional scans were very useful data for the discovery phase of CMB anisotropy research. Current progress depends upon the availability of two-dimensional maps of low Galactic foreground regions (low dust in this case) with several thousand pixels so that sampling variance is less important. In addition one can look for properties of the sky which are not predicted by theories and could be overlooked in statistical analyses. Mapping also makes it possible to catalog features for comparison to or motivation of other experiments.

Under the auspices of the NSF Center for Particle Astrophysics a collaboration consisting of groups from the University of California at Berkeley, Caltech, the University of Rome, and the IROE-CNR Florence have developed the new systems for MAXIMA and BOOMERANG. The observations thus far have been a combination of northern hemisphere flights of MAXIMA and BOOMERANG and a long duration balloon (LDB) flight of BOOMERANG from Antarctica.

To make an imager a new optical system was necessary (Figs. 3 and 4). The primary mirror for MAXIMA is a $1.3-\mathrm{m}$, off-axis, light-weight primary mirror. The primary is modulated which allows a much larger beam chop angle on the sky with less spill over and thus more pixels in the focal plane. Cold secondary and tertiary mirrors provide a cold Lyot stop and the field-of-view required for the array of 11 arcmin pixels. The geometrical aberrations in the center of the field-of-view are less than 10 arcmin. 


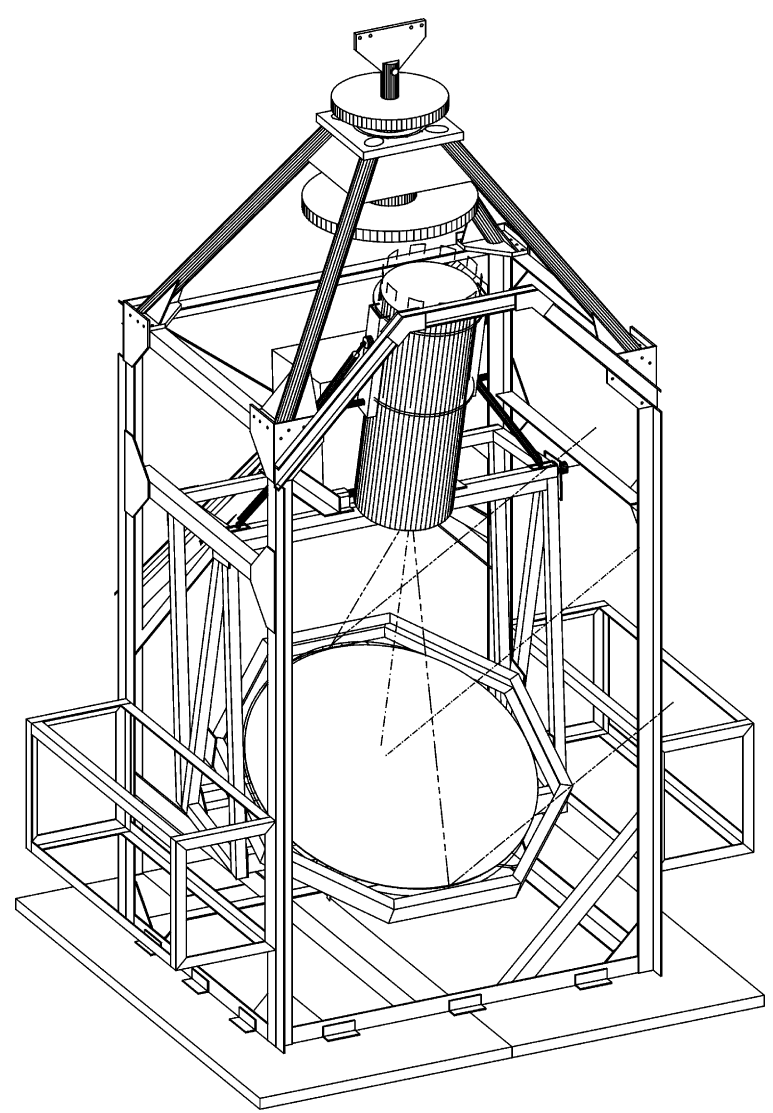

Fig. 3. A schematic drawing of the MAXIMA gondola system showing three sample rays from the sky reflecting from the nodding primary, coming to the prime focus and entering the dewar containing cold optics and bolometer detectors. Also show are the gondola frame work with the angular momentum wheels on top and two side boxes holding the electronics for the pointing system and the detector signal processing. The first stage of detector electronics is inside the dewar, the next stage in an RF shielded backpack attached to the dewar.

A larger primary mirror requires a larger gondola (Fig. 3). The chop angle can both be increased and varied allowing the instrument to sample the shape of the power spectrum over the range $40<\ell<1000$.

An additional feature is new detector electronics with AC coupling in order to allow linear scanning in a total power mode, making maps and power spectrum measurements directly. This approach is different than that of making a number of different window functions. The instrument makes a raster scan of the CMB anisotropies on the sky directly rather than obtaining a set of differences at different chop angles. One is thus mapping directly and measuring the power spectrum as the Fourier transform of the data. At this stage the instrument is designed to operate in this mode by scanning the primary mirror in a sawtooth pattern rapidly $(3 \mathrm{~Hz})$ and more slowly moving the entire gondola in azimuth to cover a larger angle.

Another major change was that from a single pixel four-frequency photometer to a 16-pixel receiver. This allows taking data at eight times the rate and thus make two-dimensional mapping feasible. The bolometers have a spider-web (silicon nitride micromesh) substrate so that cosmic ray 


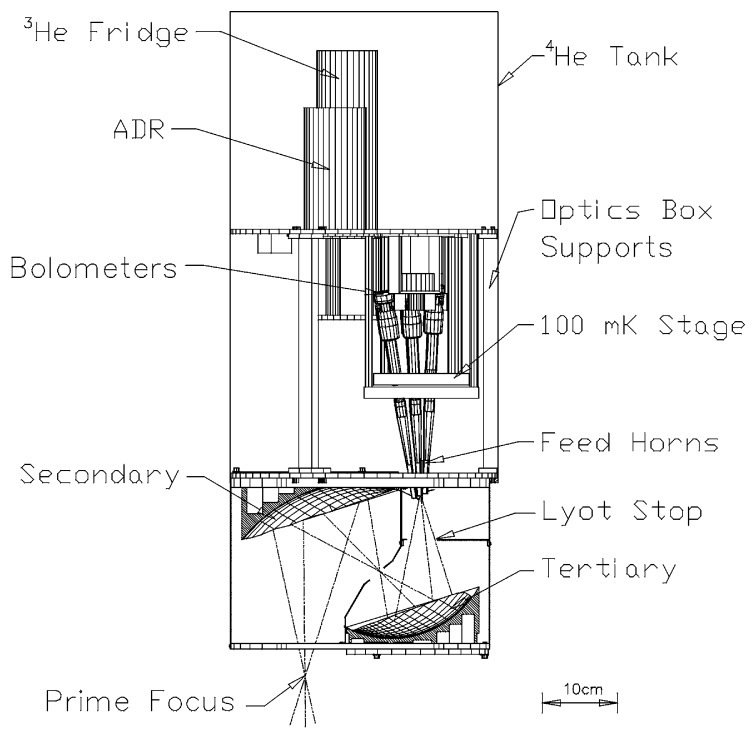

Fig. 4. The cold optical system for MAXIMA. Both MAXIMA and BOOMERANG use fast off-axis LHe-cooled secondary and tertiary mirrors to reduce optics emissions. The design incorporates cold black baffles and a cold Lyot stop which controls the illumination on the primary mirror giving smaller offsets and better control of spillover than can be achieved in optical systems that are not re-imaged. The figure also shows the location of the feedhorns and the bolometers as well as the Adiabatic Demagnitization Refrigerator (ADR) and He3 fridges used to maintain the bolometers at $100 \mathrm{mK}$. The optics are shown in cross-section for clarity.

transient occurrences will be reduced by more than an order of magnitude. The first flight of the new gondola was September 1995 and the first flight of array receiver was in August 1998. MAXIMA array receiver second flight occurred in June 1999. We can anticipate that the MAXIMA maps and the anisotropy power spectrum around the location of the first and second doppler peak will be available in the near future. Fig. 5 indicates an estimate of the accuracy of the power spectrum determination.

\subsubsection{BOOMERANG}

BOOMERANG is effectively equivalent to the long-duration balloon-borne version of MAXIMA and an intermediate step toward the bolometer space mission, the Planck HFI. BOOMERANG had a northern hemisphere flight in August 1998 followed by a 10-day flight circumnavigating Antarctica in the austral summer beginning December 29, 1998 and lasting until January 8, 1999. BOOMERANG has mapped a significant region of the sky. The BOOMERANG focal plane contains 8 pixels: four multiband photometers $\left(6,9\right.$ and $\left.14 \mathrm{~cm}^{-1}\right)$ and four monochromatic channels $\left(3 \mathrm{~cm}^{-1}\right)$. The diffraction limited angular resolution is $12^{\prime}$ above $6 \mathrm{~cm}^{-1}$ and $20^{\prime}$ at $3 \mathrm{~cm}^{-1}$. In total power mode, the largest resolution is limited only by the length of a scan (see Fig. 6).

The high cosmic-ray flux over the Antarctic requires detectors which are insensitive to cosmic rays. "Spider web" bolometers have been developed specifically to minimize the effect of cosmic rays on the detector. These bolometers are called composite because the functions of absorbing radiation and measuring the temperature increase are separated. The absorber is the "spider web" 


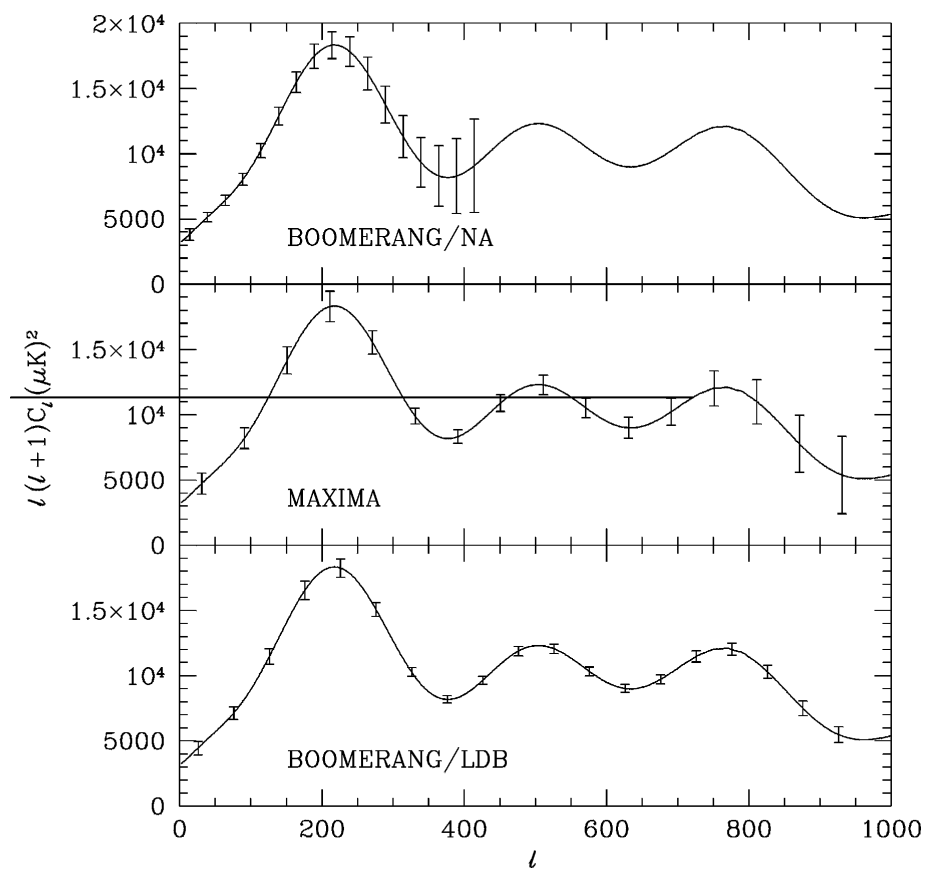

Fig. 5. Estimates of the accuracy with which each of the three flights for MAXIMA and BOOMERANG will determine the power spectrum of CMB anisotropy. The sold line in each frame represents the standard cold dark matter ( $\Omega=1, \Omega_{\mathrm{b}}=0.05, h=50$, no reionization) power spectrum. The spacing of the error flags indicates the resolution provided by each experiment; the amplitude of the error flags indicate one standard deviation uncertainty and includes the effects of instrumental noise, sky coverage, and differences strategy.

and a thermometer is a neutron transmutation doped (NTD 14) thermistor. Spider web bolometers for BOOMERANG have a noise equivalent power (NEP) of $\sim 1 \times 10^{-17} \mathrm{~W} / \mathrm{Hz}^{1 / 2}$.

The electrical circuit of BOOMERANG is split in subcircuits which reside at different temperatures. There are bolometers at $300 \mathrm{mK}$, which are AC-biased at $200 \mathrm{~Hz}$ and dual JFET source followers, providing a low impedance line going out of the cryostat. At $300 \mathrm{~K}$, the signal is preamplified, demodulated and filtered through a small bandwidth, thus enabling the detection of signals of the order of nanovolts.

The BOOMERANG North American flight produced a map covering a large arc around the North Celestial Pole. It covers about $10 \%$ of the full sky. This region was mapped by sweeping in azimuth $30^{\circ} \mathrm{N}$ latitudes while holding the elevation constant; after $12 \mathrm{~h}$, the rotation of the Earth produced a map of half of a circular region centered on the NCP.

The region mapped by the Antarctic flight is centered on the Southern Hole, a region of exceptionally low Galactic dust emission in the southern sky. During the Antarctic summer, the anti-solar direction passes through the Southern Hole; the BOOMERANG scans were centered on the antipode of the sun's path to minimize sidelobe response and thermal response from the sun.

BOOMERANG complements the DMR with high-sensitivity measurement of CMB power on scales between 12 arcmin and $\sim 10^{\circ}$. In Fig. 5, the error bars represent the $\pm 1 \sigma$ limit which 


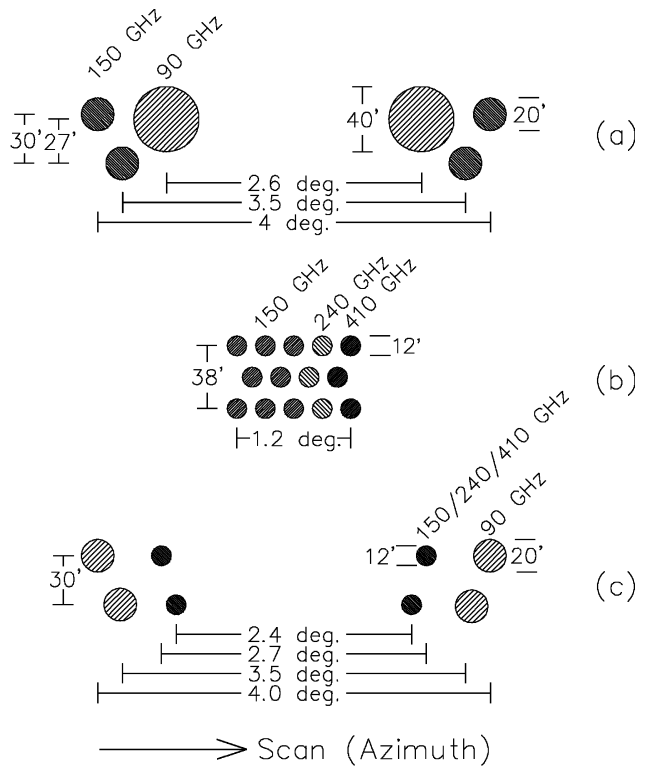

Fig. 6. Layout of the three BOOMERANG and MAXIMA focal planes. The focal planes are: (a) BOOMERANG North American flight, (b) MAXIMA, and (c) BOOMERANG LDB (Antarctica). Circles indicate the relative position and FWHM of the beams projected on the sky. Each circle represents a dual-polarization, high efficiency feed, with the exception $12^{\prime}$ beams shown in (c), which represent multi-frequency photometers of the type flown previously on MAX. Each experiment is scanned in azimuth, which is horizontal in the figure.

BOOMERANG is expected to determine for a standard cold dark matter model. The BOOMERANG-MAXIMA program will return excellent scientific data and will be a good test of the instrumentation and techniques for Planck.

\section{2. $M S A M /$ TopHat}

MSAM/TopHat is a collaboration working on a series of experiments to measure the mediumscale anisotropy of the cosmic microwave background radiation. The collaboration includes researchers at the Bartol Research Institute, Brown University, the University of Chicago, the Danish Space Research Institute, and NASA/Goddard Space Flight Center. The MSAM/TopHat program is similar to the MAX/MAXIMA/BOOMERANG program. TopHat is scheduled for launch from Antarctica in the 1999/2000 season.

\subsubsection{MSAM}

MSAM is an acronym for medium scale anisotropy measurement. A notable difference between MSAM and MAX has been that MSAM used a three-position chop analyzed either as a triple beam or double beam (two chop angles on the sky) observation. MSAM angular resolution is $0.5^{\circ}$ between 5 and $23 \mathrm{~cm}^{-1}$ (150 and $700 \mathrm{GHz}$ or wavelengths $0.4-2.0 \mathrm{~mm}$ ). MSAM has had three flights (June 1992, May 1994, June 1995) all from Palestine, Texas [16,17]. 
MSAM-I: The first phase of the medium-scale anisotropy measurement (MSAM-I) probed CMB anisotropy at $0.5^{\circ}$ angular scales between 5 and $23 \mathrm{~cm}^{-1}(150$ and $700 \mathrm{GHz}, 0.4$ and $2.0 \mathrm{~mm})$. The first flight of this package (June 1992 from Palestine, TX) has resulted in a detection of $0.5 \times 10^{-5}<\delta T / T<2 \times 10^{-5}$. Fluctuations at these angular scales are believed to be the precursors of the largest structures we observe today. This level of anisotropy is at the lower end of the predicted values from standard cold dark matter theories of structure formation. The interpretation of the results, however, is complicated by the presence of two point sources in the data. This hints at the possibility of a previously unsuspected population of objects which will challenge anisotropy measurements at these sensitivity levels. Such sources may be distinguished by the use of multiple spectral bands bracketing the peak of the CMB, such as those in MSAM-I and MSAM-II. One can also interpret the two extra peaks as CMB fluctuations and then the data are in good agreement with the anisotropy predicted by SCDM.

MSAM-II is the second phase of the medium-scale anisotropy measurement. An adiabatic demagnetization refrigerator (ADR) is used to cool monolithic silicon bolometers to $100 \mathrm{mK}$ in a new radiometer. The new radiometer has expanded frequency coverage in 5 spectral bands between 2.3 and $5 \mathrm{~cm}^{-1}(70-150 \mathrm{GHz}$ or wavelengths $2.0-4.3 \mathrm{~mm})$. The instrument is expected to improve the signal-to-noise ratio by about a factor of three over the previous results.

MSAM observations are along a ring surrounding the north celestial pole with a $20^{\prime}$ beam on the sky giving sensitivity to the medium angular-scale power spectrum of the radiation.

\subsubsection{TopHat}

TopHat is conceived as a long-duration balloon-borne experiment with the detectors located on the top of the balloon rather than in a gondola hanging below the balloon. Designed to provide reliable, quantitative measurements of the CMBR anisotropy, TopHat is optimized to reject both systematic and foreground spurious signals. By mounting the telescope on top of the balloon, the entire sky above the instrument will be free from supporting structures which could scatter radiation into the sidelobes of the optics - a critical source of systematic uncertainty for anisotropy measurements at the $10^{-6}$ level of sensitivity. Two versions of TopHat are being constructed: the Pointer and the Spinner.

The TopHat Pointer is similar to MSAM both in the Cassegrain design of the telescope and its ability to perform pointed integrations. The beam size makes this instrument sensitive to angular scales as fine as $0.33^{\circ}$. The TopHat Pointer will be able to observe the same region of sky as MSAM2 with similar sensitivity except at shorter wavelengths. This will provide unprecedented spectral coverage of a significant portion of the sky. Not only does this yield an excellent check of systematic errors which can plague any experiment of this sensitivity, it also will probe the nature of the far-infrared foreground spectrum in high galactic latitudes. This experiment flight was originally planned to fly from mid-latitudes in 1998. Following the recommendations of an independent scientific review, this flight was deferred in order to advance the schedule for the TopHat Spinner.

The TopHat Spinner will map a $48^{\circ}$ diameter disk of sky above the Southern Polar Cap at 20 arcminute resolution. To accomplish this, the telescope, tipped $12^{\circ}$ off the zenith, simply spins at a constant rate about the vertical axis. Each rotation sweeps out a circle of $24^{\circ}$. As the Earth rotates, the entire polar cap is observed each day. The TopHat Spinner will be launched from Antarctica as a long duration balloon (LDB) flight and will have approximately 2 weeks of 
observation time. This will permit a substantial fraction of the flight to be dedicated to studying and characterizing systematics in-flight while still maintaining high sensitivity to CMBR anisotropy. Observing in five spectral bands between 5 and $21 \mathrm{~cm}^{-1}$ (frequency ranges 150 and $630 \mathrm{GHz}$, or wavelength ranges $0.5-2.0 \mathrm{~mm}$ ) with anisotropy sensitivity on 0.33 to 48 degree angular scales, a single TopHat Spinner flight is expected to measure over 1800 square degrees of sky and about 20000 independent points.

The TopHat Spinner is expected to be launched from Antarctica in late 1999 or 2000. The components in a series of test flights in 1998 and 1999.

This integrated program reflects a central lesson underscored by the COBE/DMR experience - that extremely careful understanding of systematic errors and foreground sources, and not simply raw detector sensitivity, is the key to any unequivocal measurement of the CMBR anisotropy.

The extended observation time ( $\sim 2$ weeks) made possible by LDB will permit a substantial fraction of the flight to be dedicated to studying and characterizing systematics in-flight while still maintaining high sensitivity to CMB anisotropy.

\section{3. $A C E / B E A S T$}

As a follow up to their South Pole HEMT observations the Santa Barbara group has proposed Advanced Cosmic Explorer (ACE). It is a large, light-weight $(200 \mathrm{~kg})$, system aimed at making flights lasting 90 days or more. They plan to utilize advanced HEMTs, active refrigerators, and a 2-m diameter mirror to cover the frequency range $25-90 \mathrm{GHz}$. In three such flights such a system could map $75 \%$ of the sky to an angular resolution of 10 arcminutes at a level of about $20 \mu \mathrm{K}$. This project is still in the early phase but is indicative of what with sufficient funding one might achieve by the year 2000 .

\subsection{Ground-based instruments}

Ground-based instruments have provided significant CMB anisotropy observations. They have been successful as a result of the observers' clever strategies to minimize and reduce the effect of the atmosphere. These strategies have included going to high, dry sites such as the South Pole and Teide peak on Tenerife and using triple-beam chopping or other similar techniques. These techniques are more difficult to use when going to mapping and making observations over an extended portion of the power spectrum. Here again it is possible that significant progress can be made though it is likely to be eventually limited before the science is exhausted.

An exciting exception is the use of aperture synthesis interferometers. The Ryle Telescope images of the Sunyaev-Zeldovich effect in clusters and the Cambridge Anisotropy Telescope (CAT) results have convinced many that interferometers have a bright future in actually mapping anisotropy on small angular scales over selected regions of the sky.

\subsubsection{CAT: Cambridge Anisotropy Telescope; $30^{\prime}$ to $2^{\circ}$}

The CAT [67] is a three-element interferometer which can operate at frequencies between 13 and $17 \mathrm{GHz}$ with a bandwidth of $500 \mathrm{MHz}$. This frequency range was chosen as a compromise between the effects of atmospheric emission, which increase with frequency, and Galactic synchrotron and 
bremsstrahlung emission, which decrease with frequency. The most important contaminating signal for the CAT is that from discrete extragalactic radio sources. The observation strategy is to chose fields with minimum source content and then observe the sources with the higher resolution Ryle Telescope at $15.7 \mathrm{GHz}$.

The CAT has a system temperature of approximately $50 \mathrm{~K}$. Variations in the system temperature are continuously measured using a modulated $1-\mathrm{K}$ noise signal injected into each antenna. The interferometer baselines can be varied from 1 to $5 \mathrm{~m}$, and are scaled to give the same synthesized beam at different frequencies. The antennas have a primary beam FWHM of $2.2^{\circ}$ at $15 \mathrm{GHz}$. The CAT simultaneously records data from the two orthogonal linear polarizations. Its altitudeazimuth mount causes the plane of polarization to rotate on the sky as the telescope tracks a given field.

The CAT is situated within a 5-m high earth bank which is lined with aluminium. This shielding reduces the effect of spillover and terrestrial radio interference, but limits observations to elevations above $25^{\circ}$. The control hut is located about $100 \mathrm{~m}$ away. Each element of the telescope is a corrugated-conical horn with a parabolic reflector. The horns are mounted on a single turntable which can track in azimuth. Each antenna has an individual elevation drive. Preliminary tests have shown that crosstalk, correlator offsets, and antenna shadowing - particular problems associated with interferometers - do not affect the performance of the CAT at elevations greater than $40^{\circ}$ $[68,59]$. Results $[38,72]$ are shown in Fig. 2 summarizing anisotropies.

\subsubsection{Interferometers: VSA, CBI, and DASI}

Three major interferometer projects are funded and underway. They are the VSA (Very Small Array, $15^{\prime}$ to $4^{\circ}$ ) in England, the Caltech interferometer CBI (Cosmic Background Interferometer, $4^{\prime}$ to $20^{\prime}$ ) and the University of Chicago DASI (Degree Angular Scale Interferometer, $15^{\prime}$ to $1.4^{\circ}$ ) (see Table 2). These interferometers are likely to provide a very good first look at the CMB anisotropy power spectrum on angular scales less than about $0.5^{\circ}(\ell>200)$.

Table 2

Characteristics of next generation interferometers

\begin{tabular}{llll}
\hline Initials & VSA & DASI & CBI \\
Name: & Very Small Array & Very Compact Array & $\begin{array}{l}\text { Cosmic } \\
\text { Background Imager }\end{array}$ \\
\hline Frequency $(\mathrm{GHz})$ & $28-37$ & & $26-36$ \\
$N_{a}$ & 14,15 & $26-36$ & 13 \\
No. of channels & 1 tunable & 13 & 10 \\
$T_{\text {sys }}(\mathrm{K})$ & $\sim 25$ & 10 & $\sim 25$ \\
$\Delta v$ & 1.75 & $\sim 25$ & 1 \\
$\ell$ range & $150-1600$ & $150-750$ & $400-3500$ \\
Resolution & $15^{\prime}$ & $15^{\prime}$ & $4.5^{\prime}$ \\
Site & Tenerife & Antarctica & California, Chile \\
Point sources & Ryle and Bonn & A. T. & VLA and 40-m \\
Correlations & analog & analog & analog \\
Operational & 2000 & 2000 & 2000
\end{tabular}


VSA is a joint project between the Mullard Radio Astronomy Observatory (Cambridge), the Nuffield Radio Astronomy Laboratories (Jodrell Bank, Manchester), and the Instituto de Astrofisical de Canarias (Tenerife). The VSA is similar in design to CAT which was a prototype for VSA. The VSA will have 15 antennas and a 2-GHz bandwidth, analog correlators, and other technology operated on CAT. The operating frequency is $26-36 \mathrm{GHz}$ which is set by the atmospheric window and the natural waveguide bands for which high sensitivity HEMT amplifiers have been developed. The increase in frequency from CAT to VSA will also decrease the effect of discrete radio sources and Galactic emission. The atmospheric emission fluctuations will increase so that VSA will be operated on Mt. Teide on the island of Tenerife. The VSA will operate with two sets of horns: one set with a $15-\mathrm{cm}$ aperture giving a $4^{\circ}$ field of view and the second with a $30-\mathrm{cm}$ aperture giving a $2^{\circ}$ field of view. The baselines and thus resolution will scale proportionally to maintain about a $1 \mu \mathrm{K}$ sensitivity per resolution element. The VSA will get about 10 independent points of the anisotropy power spectrum with resolution of $\Delta \ell=100$ at low $\ell$ and $\Delta \ell=200$ at high $\ell$ covering the range $130<\ell<1800$.

The CBI and DASI are planning to observe the same portion of sky in the southern hemisphere. The proposed DASI is expected to image about 2500 square degrees around the South Pole region and if that goes well continued operation to cover eventually about $25 \%$ of the sky. The DASI interferometer consists of 13 scalar feed horns arranged in a closed-packed configuration which fill about $50 \%$ of the aperture to provide maximum brightness sensitivity. The horns feed low-noise HEMT amplifiers operating at $26-36 \mathrm{GHz}$ with noise temperatures of about $10 \mathrm{~K}$. The estimated sensitivity is $4-10 \mu \mathrm{K}$ in pixels ranging from $0.25^{\circ}$ to $1.4^{\circ}$ in the $3^{\circ}$ field of view. The DASI will be operated from the South Pole Station and is scheduled for installation in the fall of 1998 with first results expected the following spring.

The sensitivity of an interferometer system can be estimated using the following formulae for flux density and temperature:

Flux density

$$
\Delta S_{\mathrm{rms}}=\frac{2 k T_{\mathrm{sys}}}{\eta_{a} A_{a} \eta_{c}\left[n_{a}\left(n_{a}-1\right) \Delta v \tau\right]^{1 / 2}} .
$$

Temperature

$$
\Delta T_{\mathrm{rms}}=\frac{\lambda^{2} T_{\mathrm{sys}}}{\theta_{s}^{2} \eta_{a} A_{a} \eta_{c}\left[n_{a}\left(n_{a}-1\right) \Delta v \tau\right]^{1 / 2}} .
$$

Same expressions evaluated with typical numbers:

Flux density

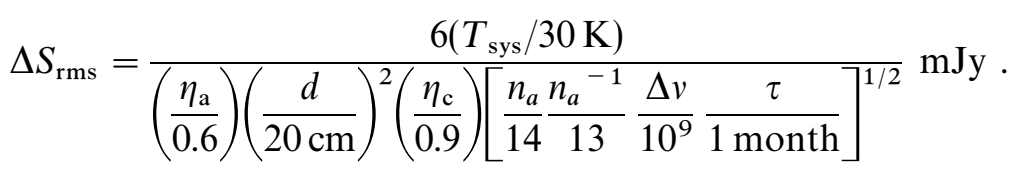

Temperature

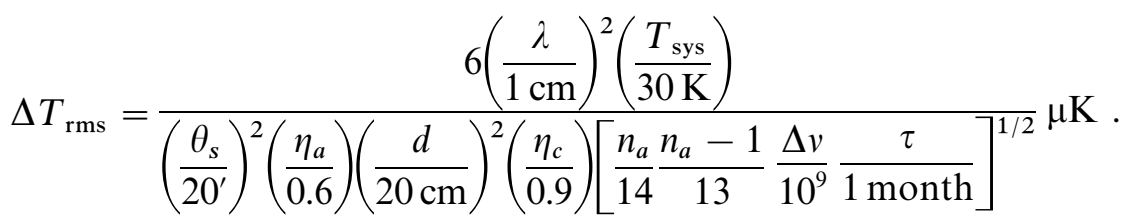


One can then evaluate these formulae and compare with Table 2 to estimate the sensitivity and sky area that can be surveyed in a given observing time and see that on small angular scales interferometers are competitive with many other experiments.

\section{5. $A C B A R$}

The Arcminute Cosmology Bolometer Array Receiver is the latest of the non-interferometer ground-based experiments. ACBAR is a 16 element $300 \mathrm{mk}$ bolometer array designed specifically for observations of CMB anisotropy and the Sunyaev-Zeldovich effect. It will operate at $4 \mathrm{~mm}$ wavelength atmospheric windows. Extending the spectral range over which observations of CMB anisotropies are made is necessary in order to separate primary anisotropies from foreground contamination. Multifrequency observations will make it possible to measure cluster peculiar velocities as well as perform sensitive searches for distant clusters of galaxies. Because of the altitude and lack of water vapor, the South Pole is presently the best terrestrial site from which to carry out this program of observations.

To observe the entire interesting region of the CMB power spectrum, one needs to have sensitivity to a large range of angular scales. The Viper telescope has a $2 \mathrm{~m}$ diameter primary mirror which gives a diffraction limited beam size at $150 \mathrm{GHz}$ of $4^{\prime}$ FWHM. Viper has a chopping tertiary at an image of the primary which will sweep the beams about $3^{\circ}$ across the sky in a second. This range of scales will give us power spectrum sensitivity on multipole moments from 160 to 2700 . To improve foreground removal, the beam sizes on the sky will be the same for all four frequencies; the four pixels in each row see the same patch of sky as they are swept in azimuth. The feeds under illuminate the primary at the higher frequencies to give matched $4^{\prime}$ corrugated scalar feeds are being used to obtain low sidelobes and cross polarization of the beams.

ACBAR's frequency coverage on small angular scales will be very complementary to the lower frequency and smaller angular scale measurements than made by the MAP satellite. In addition, combination of ACBAR SZ maps with X-ray data from the Chandra (AXAF) and XMM satellites will provide an powerful probe of the structure of massive galaxy clusters.

\section{Future satellite missions}

An accurate, extensive imaging of CMB anisotropies with sub-degree angular resolution would provide decisive answers to several major open questions on structure formation and cosmological scenarios. The observational requirements of such an ambitious objective can be met by a space mission with a far-earth orbit and instruments based on state-of-the-art technologies.

While balloon-borne and ground-based observations can do a credible job in measuring the CMB anisotropy power spectrum, atmospheric disturbance, emission from the Earth and limited integration time are the main limiting factors which prevent ground-based and balloon-borne experiments from obtaining sufficient sensitivity over very large sky regions, with additional difficulties in reaching accurate foreground removal (see Danese et al., 1995 for a recent discussion). Only a suitably designed space mission can meet the scientific goals sought by cosmologists. On the other hand, it should be stressed that experiments from the ground or from balloons are not alternative to a space mission like Planck, but rather complementary. 


\section{1. $M A P$}

Microwave anisotropy probe (MAP) was selected by NASA in 1996 as a MidEX class mission. Its launch is expected to be roughly 2001. The goal of MAP is to measure the relative CMB temperature over the full sky with an angular resolution of $0.3^{\circ}$, a sensitivity of $20 \mu \mathrm{K}$ per $0.3^{\circ}$ square pixel, and with systematic effects limited to $5 \mu \mathrm{K}$ per pixel. Details about the major aspects of the mission design are given below.

\subsubsection{Galactic emission foreground}

Galactic foreground signals are distinguishable from CMB anisotropy by their differing spectra and spatial distributions.

Fig. 7 shows the estimated spectra of the Galactic foreground signals and a range of expected cosmological signal intensities. The three physical mechanisms that contribute to the Galactic emission are synchrotron radiation, free-free radiation, and thermal radiation from interstellar dust. Results from CMB and other measurements show that at high Galactic latitudes CMB anisotropy dominates the Galactic signals in the range $30-150 \mathrm{GHz}$. However, the Galactic foreground will need to be measured and removed from some of the MAP data.

There are two techniques that MAP will use to evaluate and remove the Galactic foreground. The first uses existing Galactic maps at lower (radio) and higher (far-infrared) frequencies as foreground emission templates. Uncertainties in the original data and position-dependent spectral index variations introduce errors with this technique. There is no good free-free emission template because there is no frequency where it dominates the microwave emission. High-resolution, large-scale maps of $\mathrm{H}$-alpha emission will be a template for the free-free emission when they become available.

The second technique is to form linear combinations of multi-frequency MAP observations such that signals with specified spectra are cancelled. The linear combination of multi-frequency data makes no assumptions about the foreground signal strength or spatial distribution, but requires knowledge of the spectra of the foregrounds. Both techniques were successfully employed by COBE.

The contamination from extragalactic radio sources is not yet a solved issue. Flat spectrum radio sources observed with a diffraction limited system produce a signal with very nearly the same frequency dependence as the CMB anisotropy making the spectral shape technique ineffective. It will be necessary to compile a list of significant radio sources and excise them from the data or find another approach.

Five frequency bands with comparable sensitivity are desirable to solve for the four signals (synchrotron, free-free, dust, and CMB anisotropy) and the fifth degree of freedom is used to maximize signal to noise. The range of frequency coverage is more important than the specific choice of frequencies within the range. The lowest frequency to survey from space should be at the $22 \mathrm{GHz}$ atmospheric water line since frequencies below this can (with difficulty) be accurately measured from the ground. The highest frequency to survey should be about $100 \mathrm{GHz}$ to reduce the dust contribution and minimize the number of competing foreground signals. The choice of frequencies between 22 and $100 \mathrm{GHz}$ can be dictated by the practical consideration of standard waveguide bands. Based on these considerations, MAP has selected the five frequency bands, which are indicated in Fig. 7 and in Table 3. 


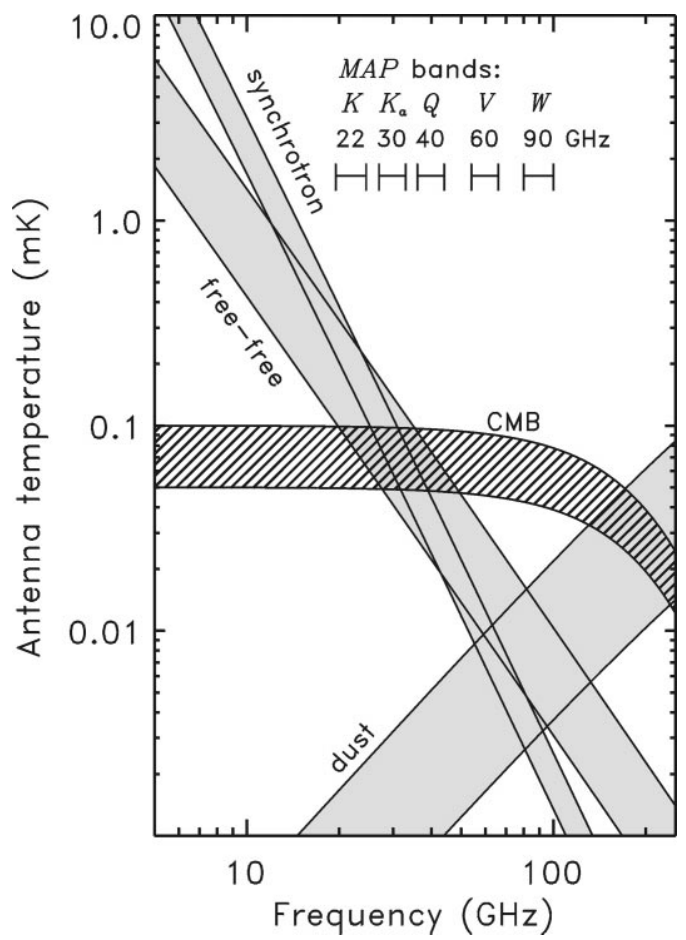

Fig. 7. Galactic foreground emission estimates plotted as a function of frequency. The width of the band indicates the estimated range for Galactic latitudes varying between $20^{\circ}<b<70^{\circ}$. The proposed MAP frequency bands are indicated.

Table 3

MAP angular resolution

\begin{tabular}{lllllll}
\hline $\begin{array}{l}\text { Frequency } \\
(\mathrm{GHz})\end{array}$ & Band name & $\begin{array}{l}\text { Wavelength } \\
(\mathrm{mm})\end{array}$ & $\begin{array}{l}\text { Beam spec } \\
\text { (deg.) }\end{array}$ & $\begin{array}{l}\text { FWHM design } \\
\text { (deg.) }\end{array}$ & $\begin{array}{l}\text { No. of } \\
\text { channels }\end{array}$ & $\begin{array}{l}\text { Sensitivity } \\
0.3^{\circ} \times 0.3^{\circ} \text { pixel }\end{array}$ \\
\hline 22 & K band & 13.6 & 0.90 & 0.93 & 4 & $35 \mu \mathrm{K} 26 \mu \mathrm{K}$ \\
30 & Ka band & 10.0 & 0.65 & 0.68 & 4 & $35 \mu \mathrm{K} 32 \mu \mathrm{K}$ \\
40 & Q band & 7.5 & 0.53 & 0.47 & 8 & $35 \mu \mathrm{K} 27 \mu \mathrm{K}$ \\
60 & V band & 5.0 & 0.39 & 0.35 & 8 & $35 \mu \mathrm{K} 35 \mu \mathrm{K}$ \\
90 & W band & 3.3 & 0.29 & 0.21 & 16 & $35 \mu \mathrm{K} 35 \mu \mathrm{K}$ \\
\hline
\end{tabular}

\subsubsection{MAP mission goals}

CMB anisotropy information from current and proposed high resolution $\left(<0.3^{\circ}\right)$ measurements over limited sky regions will likely succeed from ground and balloon-based platforms. The priority for the MAP mission is to map the entire sky with $>0.3^{\circ}$ angular resolution where the cosmological return is high, and the data cannot be readily obtained in any other way. The MAP optics feature back to back $1.5-\mathrm{m}$ primary reflectors which lead to an angular resolution of $0.29^{\circ}$ in the highest frequency $(90 \mathrm{GHz})$ channel. 


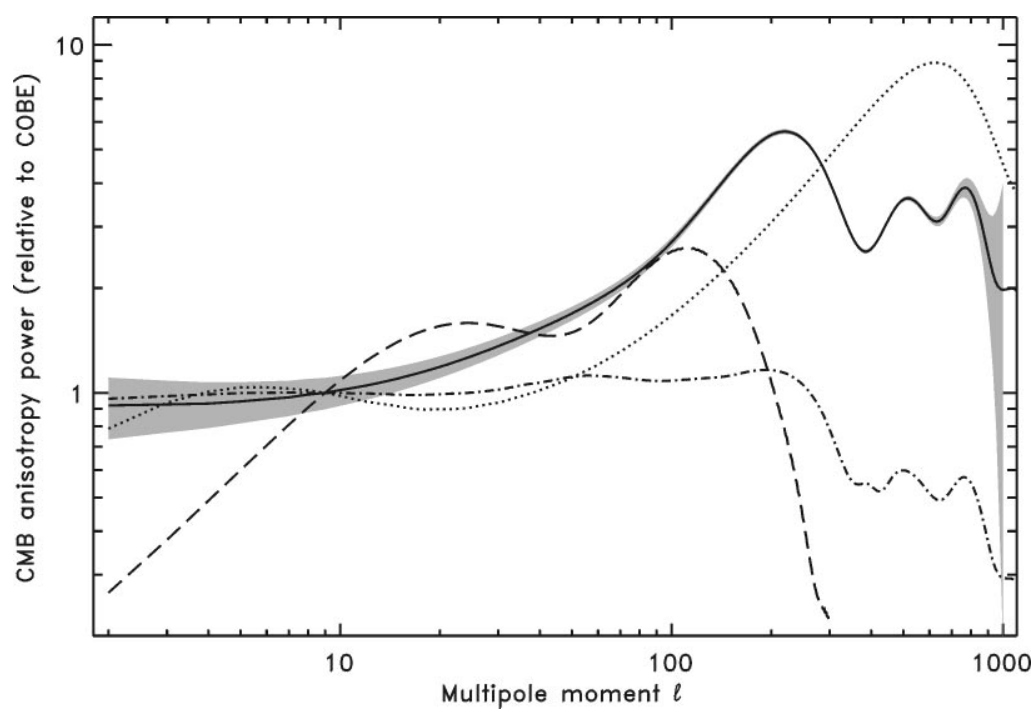

Fig. 8. Projected MAP power spectrum sensitivity. This plot shows a simple-minded estimate of the MAP sensitivity in measuring the CMB power spectrum. The gray band straddling the solid (CDM) curve indicates the MAP sensitivity after combining the three highest frequency channels and averaging the spectrum over a $10 \%$ band in spherical harmonic order. The curves plotted correspond to a standard CDM model (solid), a highly reionized CDM model (dot-dash), an open CDM model (dotted), and a primordial baryon isocurvature (PBI) model (dashed).

Table 3 gives the angular resolution to be obtained from each of the five MAP frequency bands. The value quoted is the full width at half maximum (FWHM) of the approximately Gaussian central beam lobe, in degrees.

The MAP specification calls for an equal noise sensitivity per frequency band of $35 \mu \mathrm{K}$ per $0.3^{\circ} \times 0.3^{\circ}$ square pixel. The mission duration required to meet this specification is one year of continuous observation. If Galactic emission is negligible at high latitudes above $40 \mathrm{GHz}$, as was the case for COBE, the sensitivity achievable by combining the three highest frequency channels is $20 \mu \mathrm{K}$ per $0.3^{\circ} \times 0.3^{\circ}$ pixel.

The corresponding sensitivity to the angular power spectrum, obtained with simple analytic formulae, is illustrated in Fig. 8 which shows the predicted power spectra for a number of competing structure formation models. The gray band straddling the solid (CDM) curve indicates the MAP sensitivity after combining the three highest frequency channels and averaging the spectrum over a $10 \%$ band in spherical harmonic order.

\subsubsection{MAP trajectory and orbit}

To minimize environmental disturbances and maximize observing efficiency, MAP will observe from a Lissajous orbit about the L2 Sun-Earth Lagrange point 1.5 million $\mathrm{km}$ from Earth. The trajectory selected to attain such an orbit consists of 2.5-3.5 lunar phasings loops followed by about a 100 day cruise to L2. No thruster firings are required to enter the L2 orbit. 
The L2 Lagrange point offers a virtually ideal location from which to carry out CMB observations. Because of its distance, 1.5 million km from Earth, it affords great protection from the Earth's microwave emission, magnetic fields, and other disturbances. It also provides for a very stable thermal environment and near 100\% observing efficiency since the Sun, Earth, and Moon are always behind the instrument's field of view.

MAP's trajectory features 2.5 or 3.5 lunar phasing loops which assist the spacecraft in reaching L2. The cruise time to L2 is approximately 100 days after the lunar phasing loops are completed. The launch window for this trajectory is about $20 \mathrm{~min} /$ day for 7 consecutive days each month. Once in orbit about L2, the satellite maintains a Lissajous orbit such that the MAP-Earth vector remains between $1^{\circ}$ and $10^{\circ}$ off the Sun-Earth vector to satisfy communications requirements while avoiding eclipses. Station-keeping maneuvers will be required about 4 times per year to maintain this orbit.

\subsubsection{MAP instrumentation}

The MAP instrument (Fig. 9) consists of two back-to-back, off-axis Gregorian telescopes that produce two focal planes, A and B, on opposite sides of the spacecraft symmetry axis. A set of 10 corrugated horn feeds lie in each focal plane and collect the signal power that goes to the amplification electronics. The microwave system consists of 10 4-channel differencing assemblies that are designed to eliminate low-frequency gain instabilities and amplifier noise in the differential signal.

The reflector design incorporates two back-to-back off-axis Gregorian telescopes with 1.5-m primary reflectors and $0.52-\mathrm{m}$ secondary reflectors. Each primary is an elliptical section of a paraboloid, while the secondaries are nearly elliptical. This arrangement produces two slightly convex focal surfaces on opposite sides of the spacecraft spin (symmetry) axis with plate scales of about $15^{\prime} / \mathrm{cm}$. The $99.5 \%$ encircled energy spot size diameter is less than $1 \mathrm{~cm}$ over a $15 \times 15 \mathrm{~cm}$ region of the focal plane, and less than $0.33 \mathrm{~cm}$ over the central $8 \times 8 \mathrm{~cm}$ region.

Diffraction shields are employed above, below, and to the sides of each secondary to limit diffracted signals to less than $0.5 \mu \mathrm{K}$. In addition, the deployable solar panels and multi-layer insulation guarantee that the secondaries remain at least $6^{\circ}$ into the shadow from the Sun during observing.

The feed design calls for as small an aperture as possible consistent with a primary edge taper requirement of $-25 \mathrm{~dB}$, and a length that places the throat of each differential feed pair in close proximity to the other. The feed aperture diameters scale inversely with frequency, while the primary is equally illuminated at each frequency, leading to a beam size inversely proportional to frequency. The feeds are corrugated to produce beams with high symmetry, low-loss, and minimal sidelobes: the extremely low loss $\mathrm{HE}_{11}$ hybrid mode dominates. The phase center of each feed is kept as close as possible to its aperture, resulting in a frequency-independent beam for each feed. Since the distance from the focal plane to the spacecraft symmetry axis is nearly the same for all the feeds, the high-frequency feeds are extended with low loss corrugated waveguide, while the low-frequency feeds are "profiled" to reduce their length, while limiting excitation of the $\mathrm{TE}_{11}$ mode to less than $-30 \mathrm{~dB}$.

The microwave system consists of 104 -channel differencing assemblies, one for each pair of feeds. One assembly operates at $22 \mathrm{GHz}$, one at $30 \mathrm{GHz}$, two at $40 \mathrm{GHz}$, two at $60 \mathrm{GHz}$, and four at 

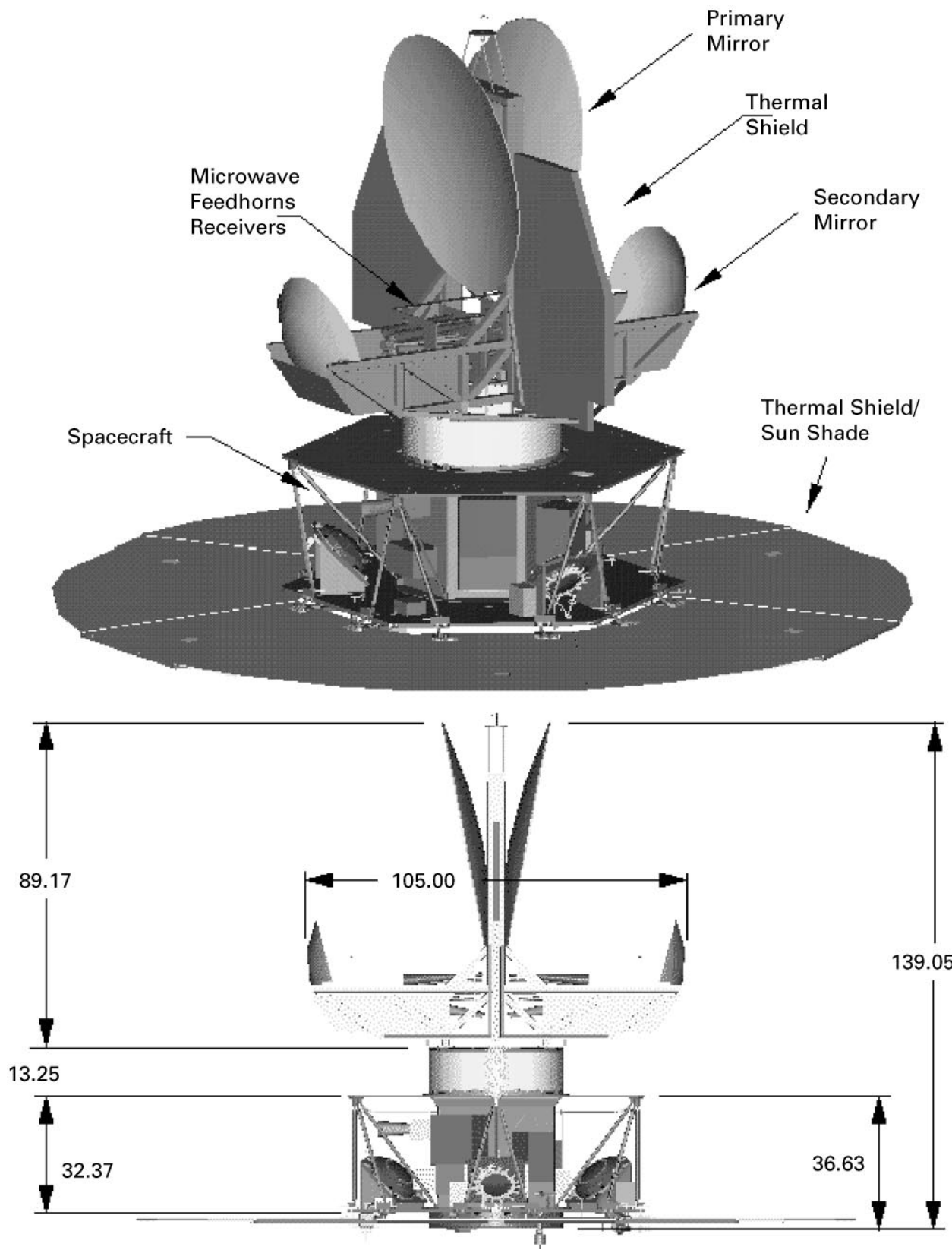

Fig. 9. Microwave anisotropy probe (MAP) artists conception shown in two views.

$90 \mathrm{GHz}$. The base of an A-side feed in the focal plane assembly (FPA) is attached to a low-loss orthomode transducer (OMT) which separates the signal into two orthogonal polarizations, A and $\mathrm{A}^{\prime}$. The A side signal is differenced against the orthogonal polarization, $\mathrm{B}^{\prime}$, from the corresponding $\mathrm{B}$-side feed, and vice versa. 
The differencing is accomplished by first combining the two signals $\mathrm{A}$ and $\mathrm{B}^{\prime}$ in a hybrid tee to form $\left(\mathrm{A}+\mathrm{B}^{\prime}\right) / \sqrt{2}$ and $\left(\mathrm{A}-\mathrm{B}^{\prime}\right) / \sqrt{2}$, then amplifying each in two cold HEMT amplifiers and sending the phase-matched outputs to the warm receiver box via waveguide. The two signals are amplified in two warm HEMT amplifiers, phase switched between $0^{\circ}$ and $+90^{\circ}$ or $-90^{\circ}$, respectively, at $2.5 \mathrm{kHz}$, then split back into $\mathrm{A}$ and $\mathrm{B}^{\prime}$ in a second hybrid tee. At this point, the two signals are square-law detected, amplified by two line drivers, and sent to the Analog Electronics Unit for synchronous demodulation and digitization. The other pair of signals, $\mathrm{A}^{\prime}$ and $\mathrm{B}$, are differenced in the same manner giving a total of four amplification channels per differencing assembly.

The splitting, phase switching, and subsequent combining of the signals enhances the instrument's performance in two ways:

(1) Since both signals to be differenced are amplified by both amplifier chains, gain fluctuations in either amplifier chain act identically on both signals and thus cancel upon differencing. (2) The phase switches introduce a $180^{\circ}$ relative phase change between the two signal paths, thereby interchanging which signal is fed to which square law detector. Thus, low-frequency noise from the detector diodes is common mode and also cancels, further reducing susceptibility to systematic effects.

\subsubsection{Map making with differential data}

MAP will observe temperature differences between points separated by $135^{\circ}$ on the sky. Maps of the relative sky temperature will be produced from the difference data by a modification of the algorithm used by COBE-DMR.

The algorithm MAP will use to reconstruct sky maps from differential data is iterative. It is mathematically equivalent to a least-squares fitting of the temperature differences to the map pixel temperatures. However, the scheme has a very intuitive interpretation: for a given pair of differential feeds, A and B, the A feed can be thought of as viewing the sky while the B feed can be thought of as viewing a comparative reference signal, or vice versa. In MAP's case, the comparative signal is a different point in the sky. The actual signal MAP measures is the temperature difference between two points on the sky, $\Delta T=T(\mathrm{~A})-T(\mathrm{~B})$, where $T(\mathrm{~A})$ is the temperature seen by feed $\mathrm{A}$, and likewise for $\mathrm{B}$. If the temperature $T(\mathrm{~B})$ is known, one could recover $T(\mathrm{~A})$ using $T(\mathrm{~A})=\Delta T+T(\mathrm{~B})$, but since $T(\mathrm{~B})$ is not known, the algorithm makes use of an iterative scheme in which $T(\mathrm{~B})$ is estimated from the previous sky map iteration. Thus, the temperature in a pixel of a map is given by the average of all observations of that pixel after correcting each observation for the estimated signal seen by the opposite feed.

For this scheme to be successful it is imperative for a given pixel to be observed with many different pixels on its ring of partners $135^{\circ}$ away. Thus the method requires a carefully designed scan strategy. The MAP strategy achieves this while simultaneously avoiding close encounters with the sun, Earth, and moon. The algorithm has been tested with the MAP scan strategy using an end-to-end mission simulation that incorporates a realistic sky signal, instrument noise, and calibration methods. The results of these simulations are described in detail in an Astrophysical Journal article [89]. After 40 iterations of the algorithm, the artifacts that remain in the map due to the map-making itself have a peak-peak amplitude of less than $0.2 \mu \mathrm{K}$, even in the presence of Galactic features with a peak brightness in excess of $60 \mathrm{mK}$. 


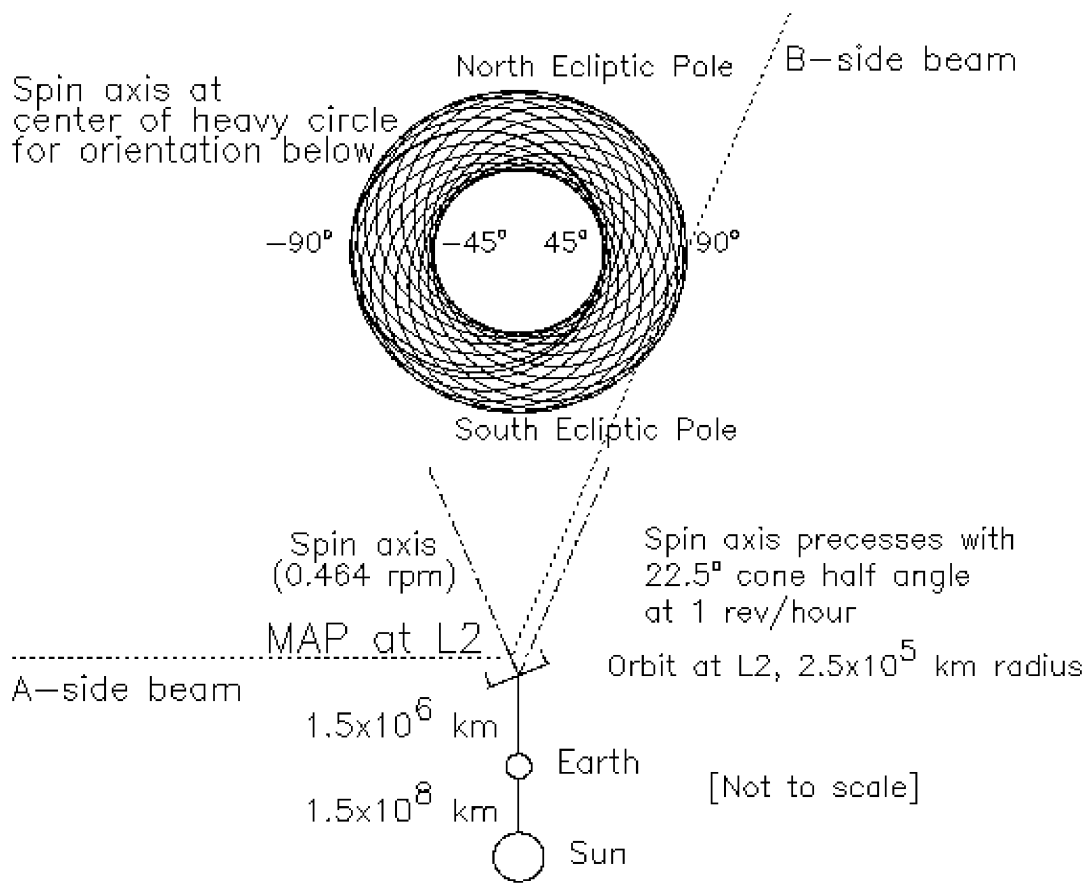

Fig. 10. The MAP scan pattern for $1 \mathrm{~h}$ of observation. The lines show the path for one side of a differential antenna pair. The other pair member follows a similar path, only delayed by $1.1 \mathrm{~min}$. There are four principal time scales for the observations. The phase of the difference signal is switched by $180^{\circ}$ at $2.5 \mathrm{kHz}$. The spacecraft spins around its symmetry axis with a $2.2 \mathrm{~min}$ period (bold circle) with cone opening angle of roughly $135^{\circ}$. This pattern precesses about the Earth-Sun line with a period of 60 minutes. Thus, in about 1 hour, over $30 \%$ of the sky is covered. Every six months, the whole sky is observed. Note that any pixel is differenced to another pixel in many directions.

\subsubsection{MAP sky coverage and scan strategy}

MAP will observe the full sky every six months (Fig. 10). The MAP scan strategy combines spacecraft spin and precession to achieve the following: (1) The MAP instrument observes more than $30 \%$ of the sky each day; (2) The spacecraft spin (and symmetry) axis maintains a fixed angle of $22.5^{\circ}$ from the sun-Earth line to mitigate systematic effects; and (3) Each sky pixel is connected to thousands of other sky pixels to ensure high-quality map solutions with negligible noise correlations.

Since a major goal of cosmology is to determine the statistical properties of the Universe, it is clear that the largest possible number of sky samples improves constraints on cosmological models. The measurement of each individual position on the sky is an independent sample of the cosmology of the universe. Moreover, full sky coverage is absolutely required to accurately determine the low-order spherical harmonic moments. While the largest angular scales were observed by COBE, MAP will remeasure the full sky with higher resolution to:

- Avoid relative calibration errors when two or more experimental results are combined (e.g., COBE and MAP).

- Provide greater sensitivity to the angular power spectrum.

- Independently verify the COBE results. 
The goals of the MAP scan strategy include the following:

- The angular separation between the two observing beams should be "large" in order to maintain sensitivity to signal at large angular scales. This is important for comparing the MAP results to $\mathrm{COBE}$, for properly normalizing the angular power spectrum, and for retaining sensitivity to the dipole which will serve as MAP's primary calibration source.

- Observe a large fraction of the sky every day. This guarantees that sky pixels will be observed at many different times in the mission which provides the capability to monitor instrument stability on many different time scales.

- Maintain a fixed angle between the spacecraft spin axis and the local solar vector. This provides for stable illumination of the spacecraft solar panels which lie normal to the spin axis, and provides a thermally stable environment to mitigate systematic effects.

- Connect each sky pixel to as many other sky pixels as possible to provide high quality map solutions from the differential data, and to render negligible pixel-pixel noise correlations. Since the MAP beam separation is fixed, this implies observing as many pixels on the differential ring of pixels as possible.

The MAP beam separation is $135^{\circ}$. Each beam axis points $67.5^{\circ}$ away from the spin and symmetry axis of the spacecraft. The spin axis will precess in a $22.5^{\circ}$ angle about the local solar vector. The combined spacecraft spin and precession will cause the observing beams to fill an annulus centered on the local solar vector with inner and outer radii of $45^{\circ}$ and $90^{\circ}$, respectively. Thus, MAP will observe more than $30 \%$ of the sky each day and will observe the ecliptic poles every day. The spin period will be $2.2 \mathrm{~min}$ while the precession period will be $1 \mathrm{~h}$. As the Earth orbits the sun, the whole observing annulus revolves with it producing full sky coverage.

The MAP mission is moving ahead quickly. Its spacecraft preliminary design review occurred in January 1997 and instrument review was held in March 1997. At that point many of the major design features were fixed and only smaller modifications will occur. However, MAP has a WWW page http://map.gsfc.nasa.gov which can be consulted for the latest information.

\subsection{Planck - the third generation space mission}

The Planck mission is the result of the merging of two proposals presented in 1993 to the European Space Agency M3 Call for Mission Ideas: COBRAS (Cosmic Background Radiation Anisotropy Satellite [55] and SAMBA (Satellite for Measurements of Background Anisotropies [66]). The COBRAS/SAMBA team completed the ESA assessment study in May 1994, and the project continued and completed the Phase A study in May 1996. COBRAS/SAMBA, renamed Planck Surveyor, has been selected to continue within the European Space Agency M3 programme.

The Planck mission is designed for extensive, accurate mapping of the anisotropy of the CMB, with angular sensitivity from sub-degree $\left(\sim 8^{\prime}-30^{\prime}\right)$ scales up to the full sky thus overlapping with the COBE-DMR maps and with signal sensitivity approaching $\Delta T / T \sim 10^{-6}$. Planck will survey the entire sky at frequencies from 30 to $850 \mathrm{GHz}(1 \mathrm{~cm} \leq \lambda \leq 350 \mu \mathrm{m})$. Its $1.5-\mathrm{m}$ passively cooled telescope will be diffraction limited at frequencies below $375 \mathrm{GHz}$. The primary science goal is a definitive measurement of the structure in the CMB on all angular scales of $10^{\prime}$ or larger. The case 
for orbital measurements of CMB anisotropy has been well made. An accurate measurement of CMB anisotropy with angular resolution of $10^{\prime}$ will revolutionize cosmology. A full description of the baseline Planck mission is available from ESTEC at http://astro.estec.esa.nl/SA-general/Projects/COBRAS/cobras.html.

Planck has been selected as ESA's next medium-scale mission (M3). Planck is scheduled to fly in 2007, several years after the MAP NASA MidEX mission. Planck is well designed to follow MAP. Using more sophisticated detector technology, Planck will have 10 times the sensitivity, 2 or 3 (depending on frequency) times the angular resolution, and 6 times the frequency coverage of MAP. This performance will allow Planck to:

1. Measure the power spectrum of the CMB with accuracy limited by cosmic variance over almost the entire range of angular frequency space in which useful cosmological information is expected.

2. Separate Galactic and extragalactic foregrounds from the CMB with high accuracy and confidence. The broad frequency coverage will allow determination of all important foreground components without any prior assumptions about their spectra. The astrophysics of the foreground is of great interest in itself and Planck will provide extremely valuable information on these astrophysical phenomenon [25].

3. Separate secondary anisotropies due to the Sunyaev-Zel'dovich effect from primary anisotropies, and measure the SZ effect with precision in thousands of clusters of galaxies. This information, combined with X-ray data, will yield an independent measurement of the Hubble constant on large scales and probe the peculiar velocity field of clusters to high redshift.

4. Separate polarization of the CMB from that in local foregrounds and measure it with precision on angular scales as small as $7^{\prime}$.

5. Survey the sky at sub-mm $(350,550$ and $850 \mu \mathrm{m})$ wavelengths that complement the wavelength coverage of SIRTF. The chance for serendipitous discovery in the Planck survey is great.

Planck has two instruments: the low-frequency instrument (LFI), based on transistor (HEMT) amplifiers, which covers the frequency range from 30 to $100 \mathrm{GHz}$; and the high-frequency instrument (HFI), based on bolometers, which covers the frequency range $90-850 \mathrm{GHz}$.

\subsubsection{Active cooling of Planck instrumentation}

Both the HEMTs and bolometers are actively cooled on Planck in order to achieve higher sensitivity. Actively cooling the HEMTs requires the HEMT amplifier chain to be broken into a low-temperature portion and a higher-temperature portion. This split reduces the thermal load on the focal plane allowing passive cooling to a significantly lower temperature (i.e., $60 \mathrm{~K}$ ) and allowing for the use of active cooling technology of a hydrogen sorption cooler. The split HEMT design and sorption cooler enable a LFI design with the following features:

- An increase in the sensitivity of the LFI by a factor of roughly five at the highest frequency over a passively cooled receiver.

- Division of the HEMT radiometers into a cold focal assembly and a room-temperature assembly. The power dissipated in the focal assembly is more than an order of magnitude lower, allowing radiative cooling of the focal assembly to a temperature of $\leq 65 \mathrm{~K}$ instead of $\sim 100 \mathrm{~K}$. 
- Active cooling of the HEMTs in the focal assembly to $\leq 20 \mathrm{~K}$. This reduces potential thermal interactions between the LFI and HFI.

\subsubsection{Planck scientific objectives}

The Planck mission will produce all-sky maps in 8 frequency bands in the range $30-800 \mathrm{GHz}$, with CMB band sensitivity $\Delta T / T \sim 10^{-6}$ per pixel. The maps will provide a detailed description of the background radiation fluctuations. Individual hot and cold regions should be identified above the statistical noise level, at all angular scales from $\lesssim 10^{\prime}$ up to very large scales, thus providing a high-resolution imaging of the last scattering surface.

The Planck maps will provide all multipoles of the temperature anisotropies from $\ell=1$ (dipole term) up to $\ell \simeq 1500$ (corresponding to $\sim 7^{\prime}$ ). It is the information contained in this large number of multipoles that can probe the various proposed scenarios of structure formation and the shape of the primordial fluctuation spectrum (for comparison, the COBE-DMR maps are limited to $\ell \lesssim 20$ ).

Table 4 compares the ability of various CMB missions to determine cosmological parameters in a model dependent but self-consistent way. The details of the calculation are not important, with two exceptions. First, $\Omega=1$ was assumed. If $\Omega$ is smaller differences in angular resolution become even more important. Second, it was assumed that confusing foregrounds were completely removed. In practice this will not be the case. The advantage that Planck's wide frequency coverage gives is therefore not reflected in the table. Nevertheless, the power of Planck in general, and the advantages of cooling the HEMTs, are immediately apparent.

The high-resolution Planck maps will provide a key test for structure formation mechanisms, based on the statistics of the observed $\Delta T / T$ distribution. The inflationary model predicts Gaussian fluctuations for the statistics of the CMB anisotropies, while alternative models based on the presence of topological defects, such as strings, monopoles, and textures, predict non-Gaussian statistics (e.g. [21]). Due to the different nature of their early history causality constrains primordial perturbations from a source such as inflation and from topological defects to have a different anisotropy power spectra particularly in the region of the "Doppler" peaks [1]. The angular resolution and sensitivity of Planck will allow discrimination between these alternatives with tests of both the power spectrum and statistics.

The proposed observations will provide an independent test for the inflationary model. Temperature anisotropies on large angular scales can be generated by gravitational waves (tensor modes, $T$ ), in addition to the energy-density perturbation component (scalar modes, $S$ ). Most inflationary models predict a well determined, simple relation between the ratio of these two components, $T / S$, and the spectral index $n[23,54]$ :

$$
n \approx 1-\frac{1}{7} \frac{T}{S}
$$

The Planck maps may verify this relationship by measuring the overall anisotropy in conjunction with Planck's measuring linear polarization components which may make it possible to differentiate scalar from tensor modes.

The mission will be able not only to test the inflationary concept but also to distinguish between various models and determine inflationary parameters. There is an extensive literature on what can be determined about inflation such as the scalar and tensor power spectra, the energy scale of 
Table 4

Uncertainties in cosmological parameters

\begin{tabular}{llll}
\hline Parameter & MAP & Planck HFI & Planck LFI $^{\mathrm{a}}$ \\
\hline$Q_{\text {rms-ps }} / 20 \mu \mathrm{K}$ & 0.23 & 0.12 & 0.14 \\
$h$ & 0.13 & 0.032 & 0.065 \\
$h^{2} \Omega_{\mathrm{b}}$ & 0.0072 & 0.0019 & 0.0036 \\
$\Omega_{\Lambda}$ & 0.67 & 0.19 & 0.33 \\
$\Omega_{v}$ & 0.38 & 0.12 & 0.28 \\
$\Omega$ & 0.11 & 0.012 & 0.029 \\
$n_{s}$ & 0.12 & 0.017 & 0.029 \\
$\tau$ & 0.35 & 0.15 & 0.20 \\
$N_{v}$ & 0.43 & 0.16 & 0.26 \\
$T_{0} \mu \mathrm{K}$ & 0.01 & 0.01 & 0.01 \\
$Y$ & 0.01 & 0.0098 & 0.01 \\
$T / \mathrm{S}$ & 0.47 & 0.17 & 0.19 \\
$Q_{\mathrm{ps}} / 0.2 \mu \mathrm{K}$ & 0.02 & 0.000021 & 0.0006 \\
$Q_{\text {diffuse }} / 20 \mu \mathrm{K}$ & 0.19 & 0.17 & 0.18 \\
\hline
\end{tabular}

${ }^{a}$ HFI based on "spider web" bolometers.

${ }^{b}$ LFI based on InP HEMTs at $20 \mathrm{~K}$.

inflation and so on (see e.g. [77,43]). Such quality measurements lead also to good observations or constraints for $\Omega_{0}, \Omega_{\mathrm{b}}, \Lambda, H_{0}$, etc. Sub-degree anisotropies are sensitive to the ionization history of the universe. In fact, they can be erased if the intergalactic medium underwent reionization at high redshifts. Moreover, the temperature anisotropies at small angular scales depend on other key cosmological parameters, such as the initial spectrum of irregularities, the baryon density of the universe, the nature of dark matter, and the geometry of the universe (see e.g. [22,39,42,71]. The Planck maps will provide constraints on these parameters within the context of specific theoretical models.

Moreover, Planck should measure the Sunyaev-Zel'dovich effect for more than 1000 rich clusters, using the higher resolution bolometric channels. This will allow a rich analysis of clusters. Combined with X-ray observations these measurements can be used to estimate the Hubble constant $H_{0}$ as a second independent determination.

\subsubsection{Foreground emissions}

In order to obtain these scientific goals, the measured temperature fluctuations need to be well understood in terms of the various components that add to the cosmological signal. In fact, in addition to the CMB temperature fluctuations, foreground structures will be present from weak, unresolved extragalactic sources and from radiation of Galactic origin (interstellar dust, free-free and synchrotron radiation). The Planck observations will reach the required control on the foreground components in two ways. First, the full sky coverage will allow accurate modeling of these components where they are dominant (e.g. Galactic radiation near the Galactic plane). Second, the observations will be performed in a very broad spectral range (see Fig. 11).

The Planck channels will span the spectral region of minimum foreground intensity (in the range $50-300 \mathrm{GHz}$ ), but with enough margin at high and low frequency to monitor "in real-time" the 


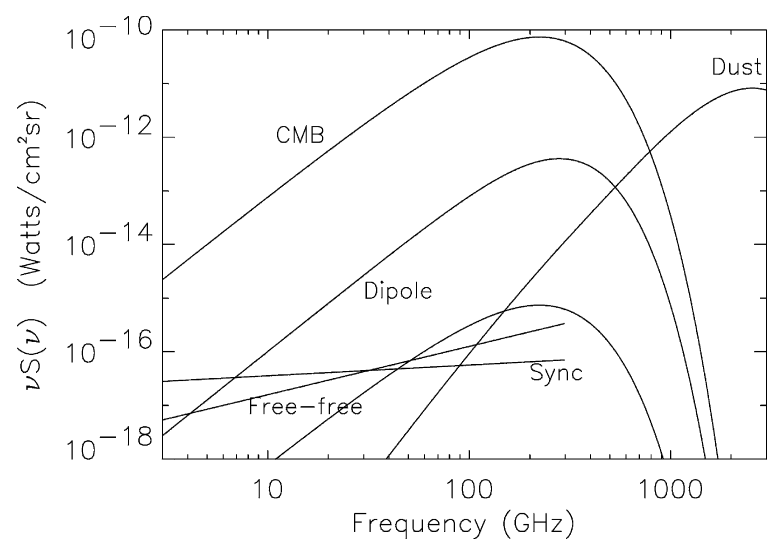

Fig. 11. The intensity of the microwave sky from 3 to $3000 \mathrm{GHz}$ near a Galactic latitude of $b=20^{\circ}$. The ordinate is the brightness of the sky times the frequency, which means the plot indicates the distribution of power per unit bandwidth. For synchrotron emission $S(v) \propto v^{-0.7}$; for free-free emission $S(v) \propto v^{-0.1}$; and for dust emission near $100 \mathrm{GHz}$ $S(v) \propto v^{3.7}$. The scaling in effective temperature is $T(v) \propto v^{-2} S(v)$. The lowest Planck-like curve is for $T=27 \mu \mathrm{K}$ or anisotropy at the $10^{-5}$ level.

effect of the various foreground components. By using the Planck spectral information and modeling the spectral dependence of Galactic and extragalactic emissions it will be possible to remove the foreground contributions with high accuracy.

It should be noted that in most channels the ultimate limitation to the cosmological information of high-quality CMB maps is expected to be due to the residual uncertainties in the separation of the foreground components rather than statistical noise. This explains why the overall design of Planck is highly driven by the need of achieving as large a spectral coverage as possible. Making the observations where the dominant foreground components are different will permit a powerful cross check on residual systematic errors in the CMB temperature fluctuation maps.

ESA (ESTEC) and the instrument teams maintain a baseline design for the mission, spacecraft, and instruments. Broad frequency coverage is achieved with arrays of HEMT amplifiers $(30-100 \mathrm{GHz})$ and bolometers $(100-850 \mathrm{GHz})$. The number of detectors and focal plane layout were optimized for the Planck mission. The characteristics of the proposed Planck payload are summarized in Table 5 [79].

The need of accurate characterization of all non-cosmological components, of course, brings the benefit of additional astrophysical information. The very large Planck data base, particularly when combined with the IRAS survey and FIRST mission, can provide information on several noncosmological issues, such as the evolution of starburst galaxies, the distribution of a cold-dust component, or the study of low-mass star formation.

\subsubsection{The payload}

The Planck payload consists of a shielded, off-axis Gregorian telescope, with a parabolic primary reflector and a secondary mirror, leading to an integrated instrument focal plane assembly. The payload is part of a spinning spacecraft, with a spin rate of $1 \mathrm{rpm}$. The focal plane assembly is 
Table 5

Characteristics of proposed Planck payload

\begin{tabular}{|c|c|c|c|c|c|c|c|c|}
\hline Inst. & $\begin{array}{l}\text { Freq. } \\
\text { (GHz) }\end{array}$ & $\begin{array}{l}\text { Angular } \\
\text { resol. }\end{array}$ & $\begin{array}{l}\text { Detector } \\
\text { device }\end{array}$ & $\begin{array}{l}T \\
(\mathrm{~K})\end{array}$ & $\begin{array}{l}\text { No. of } \\
\text { detectors }\end{array}$ & $\begin{array}{l}\text { Bandwidth } \\
(\Delta v / v)\end{array}$ & Sensitivity $^{\mathrm{a}}$ & $\begin{array}{l}\text { Linear } \\
\text { polar. }\end{array}$ \\
\hline LFI & 30 & $33^{\prime}$ & HEMT & $\sim 20$ & 4 & 0.2 & 1.6 & Yes \\
\hline LFI & 44 & $23^{\prime}$ & HEMT & $\sim 20$ & 6 & 0.2 & 2.4 & Yes \\
\hline LFI & 70 & $14^{\prime}$ & HEMT & $\sim 20$ & 12 & 0.2 & 3.6 & Yes \\
\hline LFI & 100 & $10^{\prime}$ & HEMT & $\sim 20$ & 34 & 0.2 & 4.3 & Yes \\
\hline HFI & 100 & $10.7^{\prime}$ & Bol & 0.1 & 4 & 0.25 & 1.7 & No \\
\hline HFI & 143 & $8.0^{\prime}$ & Bol & 0.1 & 12 & 0.25 & 2.0 & Yes \\
\hline HFI & 217 & $5.5^{\prime}$ & Bol & 0.1 & 12 & 0.25 & 4.3 & Yes \\
\hline HFI & 353 & $5.0^{\prime}$ & Bol & 0.1 & 6 & 0.25 & 14.4 & No \\
\hline HFI & 545 & $5.0^{\prime}$ & Bol & 0.1 & 8 & 0.25 & 147.0 & Yes \\
\hline HFI & 857 & $5.0^{\prime}$ & Bol & 0.1 & 6 & 0.25 & 6670 & No \\
\hline
\end{tabular}

${ }^{\text {a Average }} \Delta T / T$ per resolution element (12 months of observation, $1 \sigma, 10^{-6}$ units).

divided into low-frequency (LFI) and high-frequency (HFI) instrumentation according to the technology of the detectors. Both the LFI and the HFI are designed to produce high-sensitivity, multifrequency measurements of the diffuse sky radiation. The LFI will measure in four bands in the frequency range $30-100 \mathrm{GHz}(2.3-10 \mathrm{~mm}$ wavelength). The HFI will measure in four channels in the range $140-800 \mathrm{GHz}(0.4-2.1 \mathrm{~mm}$ wavelength). The highest frequency LFI channel and the lowest HFI channel overlap near the minimum foreground region. Table 5 summarizes the main characteristics of the Planck payload.

\subsubsection{The main optical system}

A clear field of view is required for a high-sensitivity CMB anisotropy experiment to avoid spurious signals arising from diffraction and reflection from the mirrors or from supports and mechanical mounting (Fig. 12). The off-axis Gregorian configuration has a primary parabolic mirror of $1.5-\mathrm{m}$ diameter, and an elliptic secondary mirror $(0.57-\mathrm{m})$. Stray radiation is minimized by underilluminating the low-emissivity optics. The telescope reimages the sky onto the focal plane instrument located near the payload platform. The telescope optical axis is offset by $85-90^{\circ}$ from the spin axis. Thus at each spacecraft spin rotation the telescope pointing direction sweeps a large (approaching a great) circle in the sky, according to the sky scan strategy.

A large, flared shield surrounds the entire telescope and focal plane assembly, to screen the detectors from contaminating sources of radiation. The shield also plays an important role as an element of the passive thermal control of the spacecraft.

\subsubsection{The focal plane assembly}

The wide spectral range requires the use of two different technologies, bolometers and coherent receivers, incorporated in a single instrument. Both technologies have shown impressive progress in the last decade, and more is expected in the near future. The thermal requirements of the two 


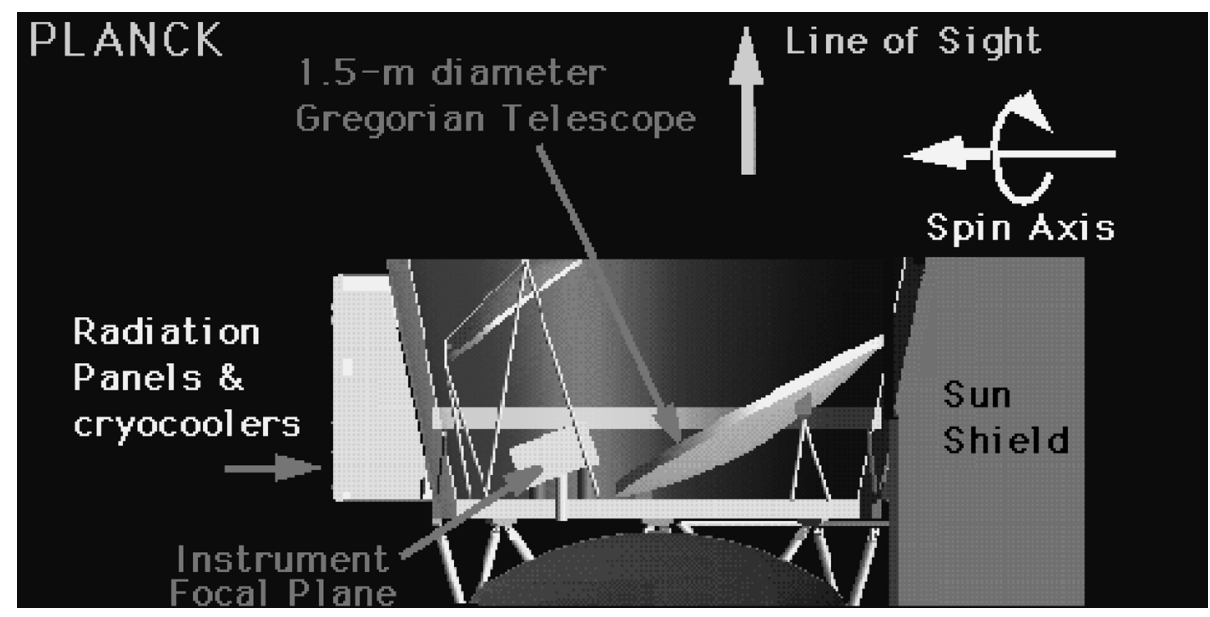

Fig. 12. Artist's concept of one possible configuration of the Planck Surveyor optics and focal plane layout.

types of detectors are widely different. The coherent radiometers (LFI), operating in the lowfrequency channels, give good performance at and operational temperature of $\leq 100 \mathrm{~K}$. Splitting the HEMT chain into cool and warmer portions leads to a passive cooling temperature of $>65 \mathrm{~K}$ and with active cooling $20 \mathrm{~K}$. The bolometers, on the other hand, require temperatures $\leq 0.15 \mathrm{~K}$ in order to reach their sensitivity performance. The main characteristics of the LFI and HFI are summarized in Table 5.

The LFI consists of an array of 26 corrugated, conical horns, each utilized in two orthogonal polarization modes, feeding a set of high sensitivity receivers. The receivers will be based on monolithic microwave integrated circuits (MMIC) technology with high electron mobility transistor (HEMT) ultra-low noise amplifiers. Since the whole LFI system will be passively cooled to the first level and then actively cooled with a sorption cooler, it can be operated for a duration limited only by spacecraft consumables ( $\sim 5$ years).

The HFI instrument will employ about 50 bolometers, operating at $\sim 0.1 \mathrm{~K}$. The cooling system combines active coolers reaching $4 \mathrm{~K}$ with a dilution refrigeration system working at zero gravity. The refrigeration system includes two pressurized tanks of ${ }^{3} \mathrm{He}$ and ${ }^{4} \mathrm{He}$ for an operational lifetime of 2 years.

\subsubsection{Orbit and sky observation strategy}

The Planck mission requires a far-from-Earth orbit. The requirements on residual Earth radiation are basically the same for the LFI and the HFI systems. Adopting a low-Earth orbit, such as that used by the COBE satellite, the requirement on straylight and sidelobe rejection would be a factor of $10^{13}$, which is beyond the capabilities of present microwave and sub-mm systems and test equipment. The orbit chosen for Planck is one about the L2 Lagrange point of the Earth-Sun system.

These L2 orbits are also very favorable from the point of view of passive cooling and thermal stability [29]. The spacecraft will be normally operated pointing in the anti-solar direction, with part of the sky observations performed within $\pm 40^{\circ}$ from anti-solar. 
The primary goal of the mission is to observe the full sky with a sensitivity of $10-15 \mu \mathrm{K}$ within the two year mission lifetime. Deeper observation of a limited ( $\sim 2 \%)$ sky region with low foregrounds could significantly contribute to the cosmological information. Simulations have shown that these observational objectives can be achieved simultaneously in a natural way, using the spinning and orbit motion of the spacecraft, with relatively simple schemes.

\section{Interpretation and future}

In seven years the field of $\mathrm{CMB}$ anisotropy observations and theory has made great strides. Until April 1992 all plots of CMB anisotropy showed only upper limits, except for the $\ell=1$ dipole. Now observations have begun to trace out the shape of the power spectrum and to make maps of the anisotropies. This observational program promises to deliver a wealth of new information to cosmology and to connect it to other fields. The COBE DMR full four-year data set has been the fields standard and anchor. We can expect little in the way of improvement compared to the final DMR results from future experiments on the large angular scales but scientific interest has moved to covering the full spectrum and learning what the medium and small angular scales will tell us. Already there are plots showing the CMB anisotropy spectrum related to and overlaid on the primordial density perturbation power spectrum and attempts to reconstruct the inflaton potential. These are the first steps in a new period of growth.

Table 6 gives an example of the level of sensitivity that might be achieved by the many experiments underway, planned, and approved. Nearly every group has data under analysis and is also at work on developing new experiments. Some of these are the natural extensions of the ongoing experiments. Some groups are considering novel approaches. Real long-term progress

Table 6

Projected parameter errors: assumes variation around Standard CDM

\begin{tabular}{|c|c|c|c|c|}
\hline & 1997 & BOOM/MAX & $\mathrm{MAP}^{\mathrm{a}}$ & Planck $^{\mathrm{a}}$ \\
\hline$\Omega$ & $0.01-2$ & $6 \%$ & $18 \%$ & $1 \%$ \\
\hline$\Omega_{\mathrm{b}}$ & $0.01 h^{-2}$ & $30 \%$ & $10 \%$ & $0.7 \%$ \\
\hline$\Lambda\left(\Omega_{\Lambda}\right)$ & $<0.65$ & \pm 0.10 & \pm 0.43 & \pm 0.05 \\
\hline$\Omega_{v}$ & $<2$ & \pm 0.25 & \pm 0.08 & \pm 0.03 \\
\hline$t_{0}$ & $12-18 \mathrm{Gyr}$ & 二 & - & - \\
\hline$H_{0}$ & $30-80 \mathrm{~km} / \mathrm{s} / \mathrm{Mpc}$ & $10 \%$ & $20 \%$ & $2 \%$ \\
\hline$\sigma_{8}$ & $0.5-0.6$ & $30 \%$ & $30 \%$ & $10 \%$ \\
\hline$Q$ & $20 \pm 2 \mu \mathrm{K}^{\mathrm{b}}$ & $30 \%$ & $30 \%$ & $10 \%$ \\
\hline$n_{s}$ & $1.0 \pm 0.5$ & $30 \%$ & $5 \%$ & $1 \%$ \\
\hline$\tau$ & $0.01-1$ & \pm 0.5 & \pm 0.2 & \pm 0.15 \\
\hline$T_{0}$ & $2.73 \pm 0.01^{\dagger}$ & - & - & - \\
\hline$Y$ & $0.2-0.25$ & $10 \%$ & $10 \%$ & $7 \%$ \\
\hline$T / S$ & $0.0-1$ & \pm 1.6 & \pm 0.38 & \pm 0.09 \\
\hline
\end{tabular}

\footnotetext{
${ }^{a}$ Ref. [13]. Note that parameters are not all independent, e.g., $H_{0} t_{0}=f(\Omega, \Lambda)$.
}

${ }^{\mathrm{b}}$ Indicates current precise results from CMB observations (COBE). 
depends on avoiding the potential foregrounds: fluctuations of the atmosphere, a source of noise that largely overwhelms recent advances in detector technology, and Galactic and extragalactic signals. This requires instruments having sufficient information (usually only through multifrequency observations) and observing frequencies to separate out the various components. It also means going above the varying atmosphere. Collaborations are working on long-duration ballooning instruments. Ultimately, as COBE has shown, going to space really allows one to overcome the atmospheric problem and to get data in a very stable and shielded environment. Two selected satellite mission are actively being developed. We can anticipate a steady and significant advance in observations. With the new data that are appearing, can be expected, and ultimately will come from the Planck mission we can look forward to a very significant improvement in our knowledge of cosmology.

\section{Acknowledgements}

This work was supported in part by the Director, Office of Energy Research, Office of High Energy and Nuclear Physics, Division of High Energy Physics of the U.S. Department of Energy under contract No. DE-AC03-76SF00098.

\section{References}

[1] A. Albrecht, D. Coulsen, P. Ferreira, J. Magueijo, Phys. Rev. Lett. 76 (1995) 1413, astro-ph 9505030.

[2] D.C. Alsop et al., Astrophys. J. 395 (1992) 317.

[3] R.A. Alpher, R.C. Herman, Phys. Today 41 (8) (1948) 24.

[4] A. Banday et al., Astrophys. J. 468 (1996) L85, astro-ph/9601064.

[5] A. Banday et al., Astrophys. J. 464 (1996) L1, astro-ph/9601066.

[6] C.L. Bennett et al., Astrophys. J. 464 (1996) L1, astro-ph/9601067.

[7] C.L. Bennett et al., Astrophys. J. 436 (1994) 423.

[8] C.L. Bennett et al., Astrophys. J. 414 (1993) L77.

[9] C.L. Bennett et al., Astrophys. J. 396 (1992) L7.

[10] C.L. Bennett et al., Astrophys. J. 391 (1991) 466.

[11] J. Bock et al., Proceedings of the 30th ESLAB Symposium on Submillimeter and Far-Infrared Space Instrumentation, 24-26 September 1996, ESTEC, Noordwijk, The Netherlands.

[12] J.R. Bond (1994), astrop-ph/9407044.

[13] J.R. Bond, G. Efstathiou, M. Tegmark (1997), astrop-ph/9702100.

[14] W.N. Brandt, C.R. Lawrence, A.C.S. Readhead, Pakianathan, T.M. Fiola, Astrophys. J. (2000), in press.

[15] A.C. Clapp, M.J. Devlin, J.O. Gundersen, C.A. Hagmann, V.V. Hristov, A.E. Lange, M. Lim, P.M. Lubin, P.D. Mauskopf, P.R. Meinhold, P.L. Richards, G.F. Smoot, S.T. Tanaka, P.T. Timbie, C.A. Wuensche, Astrophys. J. Lett. 433 (1994) 57-60, astro-ph/9404072.

[16] E.S. Cheng, D.A. Cottingham, D.J. Fixsen, C.A. Inman, M.S. Kowitt, S.S. Meyer, L.A. Page, J.L. Puchalla, J. Ruhl, R.F. Silverberg, Astrophys. J. 456 (1996) L71, astro-ph/9508087.

[17] E.S. Cheng et al., astro-ph/9705041.

[18] A.C. Clapp, M.J. Devlin, J.O. Gundersen, C.A. Hagmann, V.V. Hristov, A.E. Lange, M. Lim, P.M. Lubin, P.D. Mauskopf, P.R. Meinhold, P.L. Richards, G.F. Smoot, S.T. Tanaka, P.T. Timbie, C.A. Wuensche, Astrophys. J. Lett. 433 (1994) 57-60, astro-ph/9404072.

[19] L. Cayon, G.F. Smoot, Astrophys. J. 452 (1995) 487, astro-ph/9504072.

[20] A. de Oliveira-Costa, G.F. Smoot, Astrophys. J. 448 (1995) 477, astro-ph/9412003. 
[21] D. Coulson, P. Ferreira, P. Graham, N. Turok, Nature 368 (1994) 27.

[22] R. Crittenden, J.R. Bond, R.L. Davis, G. Efstathiou, P. Steinhardt, Phys. Rev. Lett. 71 (1993) 324.

[23] R.L. Davis, H.M. Hodges, G.F. Smoot, P.J. Steinhardt, M.S. Turner, Phys. Rev. Lett. 69 (1992) 1856, erratum 70 (1992) 1733.

[24] M.J. Devlin, A.C. Clapp, J.O. Gundersen, C.A. Hagmann, V.V. Hristov, A.E. Lange, M. Lim, P.M. Lubin, P.D. Mauskopf, P.R. Meinhold, P.L. Richards, G.F. Smoot, S.T. Tanaka, P.T. Timbie, C.A. Wuensche, Astrophys. J. Lett. 430 (1994) L1, astro-ph/940403.

[25] G. De Zotti, L. Toffolatti, F. Argüeso, R.D. Davies, P. Mazzotta, R.B. Partridge, G.F. Smoot, N. Vittorio, Proceedings of the Conference on 3K Cosmology, AIP, Vol. 476, Woodbury, NY, preprint, astro-ph/9902103.

[26] R.H. Dicke, P.J.E. Peebles, P.G. Roll, D.T. Wilkinson, Astrophys. J. 142 (1965) 414.

[27] A.G. Doroshkevich, V.N. Lukash, I.D. Novikov, The isotropization of homogeneous cosmological models, Zh. Eksper. Teor. Fiz. 64 (1973) 739-746.

[28] A.G. Doroshkevich, V.N. Lukash, I.D. Novikov, Primordial radiation in a homogeneous but anisotropic universe, Astron. Zh. 51 (1974) 554-560.

[29] R.W. Farquhar, D.W. Dunham, in: Y. Kondo (Ed.), Observatories in Earth Orbit and Beyond, Kluwer, Dordrecht, 1990, p. 391.

[30] M.L. Fischer et al., Astrophys. J. 388 (1992) 242.

[31] D.J. Fixsen et al., 1996, astro-ph/9605054, Astrophys. J. (2000), in press.

[32] K. Ganga et al., Astrophys. J. 410 (1993) L57.

[33] K.M. Górski et al., Astrophys. J. 464 (1996) L11, astro-ph/9601063.

[34] E.R. Harrison, Phys. Rev. D 1 (1970) 2726.

[35] M.G. Hauser, P.J.E. Peebles, Astrophys. J. 185 (1973) 757.

[36] G. Hinshaw et al., Astrophys. J. Lett. 464 (1996) L17, astro-ph/9601058.

[37] G. Hinshaw et al., Astrophys. J. Lett. 464 (1996) L25, astro-ph/9601061.

[38] M.P. Hobson, A.N. Lasenby, M. Jones, Mon. Not. R. Astron. Soc. (2000), submitted for publication.

[39] W. Hu, D. Scott, J. Silk, Astrophys. J. 430 (1994) L5.

[40] W. Hu, D. Spergel, M. White, PRD 55, (1996) 3288, astro-ph/9605193.

[41] G. Jungman, M. Kamionkowski, A. Kosowsky, D.N. Spergel, Phys. Rev. Lett. 76 (1995) 1007, astro-ph/ 9512139.

[42] M. Kamionkowski, D.N. Spergel, N. Sugyiama, Astrophys. J. 426 (1994) L1.

[43] L. Knox, Phys. Rev. D 52 (1995) 4307, astro-ph/9504054.

[44] A. Kogut et al., Astrophys. J. 401 (1992) 1.

[45] A. Kogut, A.J. Banday, C.L. Bennett, G. Hinshaw, K. Loewenstein, P. Lubin, G.F. Smoot, E.L. Wright, Astrophys. J. 433 (1994) 435.

[46] A. Kogut et al., Astrophys. J. 460 (1996) 1, astro-ph/9601066.

[47] A. Kogut et al., Astrophys. J. 464 (1996) L5, astro-ph/9601060.

[48] A. Kogut et al., Astrophys. J. 464 (1996) L29, astro-ph/9601062.

[49] A.T. Lee et al., Preprint, 1996, Appl. Phys. Lett., to appear.

[50] M.A. Lim, A.C. Clapp, M.J. Devlin, N. Figueiredo, J.O. Gundersen, S. Hanany, V.V. Hristov, A.E. Lange, P.M. Lubin, P.R. Meinhold, P.L. Richards, J.W. Staren, G.F. Smoot, S.T. Tanaka, astro-ph/9605142.

[51] C. Lineweaver et al., Astrophys. J. 436 (1994) 452, astro-ph/9403021.

[52] C. Lineweaver et al., Astrophys. J. 448 (1995) 482-487.

[53] C. Lineweaver et al., Astrophys. J. 470 (1996) 38, astro-ph/9601151.

[54] Little, Lyth, Phys. Lett. B 291 (1992) 391.

[55] N. Mandolesi, G.F. Smoot, M. Bersanelli, C. Cesarsky, M. Lachieze-Rey, L. Danese, N. Vittorio, P. De Bernardis, G. Dall'Oglio, G. Sironi, P. Crane, M. Janssen, B. Partridge, J. Beckman, R. Rebolo, J.L. Puget, E. Bussoletti, G. Raffelt, R. Davies, P. Encrenaz, V. Natale, G. Tofani, P. Merluzzi, L. Toffolatti, R. Scaramella, E. MartinezGonzales, D. Saez, A. Lasenby, G. Efstathiou, COBRAS (1993), proposal submitted to ESA M3.

[56] N. Mandolesi, M. Bersanelli, C. Cesarsky, L. Danese, G. Efstathiou, M. Griffin, J.M. Lamarre, H.U. NorgaardNielson, O. Pace, J.L. Puget, A. Raisanen, G.F. Smoot, J. Tauber, S. Volonte, Planet. Space Sci. 43 (1995) 1459 . 
[57] J.C. Mather et al., Astrophys. J. 420 (1994) 439.

[58] P. Meinhold et al., Astrophys. J. 409 (1993) L1.

[59] C. O'Sullivan et al., Mon. Not. R. Astron. Soc. (2000) submitted for publication.

[60] K. Ganga, L. Page, E. Cheng, S. Meyer, 1994, astro-ph/9404009.

[61] L. Page (1997), astro-ph/9703054.

[62] P.J.E. Peebles, Astrophys. J. 185 (1973) 413.

[63] P.J.E. Peebles, J.T. Yu, Astrophys. J. 162 (1970) 815.

[64] P.J.E. Peebles, Principles of Physical Cosmology, Princeton University Press, Princeton, 1993, p. 168.

[65] A.A. Penzias, R. Wilson, Astrophys. J. 142 (1965) 419.

[66] J.L. Puget, P. Ade, A. Benoit, P. De Bernardis, F. Bouchet, C. Cesarsky, F.X. Desert, R. Gispert, M. Griffin, M. Lachieze-Rey, J.M. Lamarre, P. De Marcillac, S. Masi, F. Melchiorri, F. Pajot, M. Rowan-Robinson, G. Serra, J.P. Torre, L. Vigroux, S. White, SAMBA (1993), proposal submitted to ESA M3.

[67] M. Robson, G. Yassin, G. Woan, D.M.A. Wilson, P.F. Scott, A.N. Lasenby, S. Kenderdine, P.J. Duffett-Smith, Astron. Astrophys. 277 (1993) 314.

[68] M. Robson, C.M.M. O’Sullivan, P.F. Scott, P.J. Duffett-Smith, Astron. Astrophys. 286 (1994) 1028.

[69] M. Robson, Ph.D. Thesis, University of Cambridge, 1994.

[70] R.K. Sachs, A.M. Wolfe, Astrophys. J. 147 (1967) 73.

[71] D. Scott, J. Silk, M. White, Science 268 (1995) 829.

[72] Scott et al., Astrophys. J. 461, L1.

[73] J. Silk, Nature 215 (1967) 1155-1156.

[74] G.F. Smoot et al., Astrophys. J. 360 (1990) 685.

[75] G.F. Smoot et al., Astrophys. J. 396 (1992) L1.

[76] G.F. Smoot, L. Tenorio, A.J. Banday, A. Kogut, E.L. Wright, G. Hinshaw, C.L. Bennett, Astrophys. J. 437 (1994) 1.

[77] P.J. Steinhardt, Proceedings of Snowmass Workshop, 1995.

[78] S.T. Tanaka, A.C. Clapp, M.J. Devlin, N. Figueiredo, J.O. Gundersen, S. Hanany, V.V. Hristov, A.E. Lange, M.A. Lim, P.M. Lubin, P.R. Meinhold, P.L. Richards, G.F. Smoot, J. Staren, Astrophys. J. 468 (1996) L81, astro$\mathrm{ph} / 9512067$.

[79] J.A. Tauber, Astrophys. Lett. Commun. (2000), in press.

[80] S.T. Tanaka et al., in preparation.

[81] M. Tegmark, E.F. Bunn, Astrophys. J. 455 (1995) 1.

[82] M. Tegmark, A.N. Taylor, A.F. Heavens, (1996), astro-ph/9603021.

[83] M. Tegmark, A. de Oliveira-Costa, D.J. Devlin, C.B. Netterfield, L. Page, E.J. Wollack (1996), astro-ph/9608019.

[84] M. White, E.F. Bunn, Astrophys. J. 443 (1995) L53, astro-ph/9510088.

[85] M. White, D. Scott, J. Silk, Ann. Rev. Astron. Astrophys. 32 (1994) 329.

[86] M. White, Phys. Rev. D 53 (1996) 3011, astro-ph/9601158.

[87] E.L. Wright et al., Astrophys. J. 396 (1992) L13.

[88] E.L. Wright et al., Astrophys. J. Lett. 464 (1996) L21, astro-ph/9601059.

[89] E.L. Wright, F. Hinshaw, C.L. Bennett, Astrophys. J. 458 (1996) L53.

[90] Ya.B. Zeldovich, Mon. Not. R. Astron. Soc. 160 (1972) 1.

\section{CMB Anisotropy Experiment References}

[91] ACBAR: Arcmin Cosmology Bolometer Array Receiver uses the VIPER telescope at the South Pole. http://cfpa.berkeley.edu/ swlh/research/acbar.html.

[92] ACE \& BEAST use HEMTs between 26 and $100 \mathrm{GHz}$ on both super-pressure and conventional long-duration balloon platforms. The finest angular resolution will be near $1 / 5^{\circ}$. http://www.deepspace.ucsb.edu/research/ Sphome.htm

[93] APACHE will observe from Dome-C on the Antarctic plateau. http://tonno.tesre.bo.cnr.it/ valenzia/APACHE/ apache.html

[94] Archeops: http://www-crtbt.polycnrs-gre.fr/archeops/Egeneral.html 
[95] ARGO. A balloon-borne bolometer based experiment. Results are reported in de Bernardis et al., Astrophys. J. (1994) 422:L33. http://oberon.roma1.infn.it/argo.htm

[96] ATCA: Australia Telescope Compact Array. An interferometer operating at $8.7 \mathrm{GHz}$ with a $2^{\prime}$ resolution produced a map that was analyzed for anisotropy. The results are reported in Subrahmanyan, R., Ekers, R.D., Sinclair, M. \& Silk, J. 1993, Mon. Not. R. Astron. Soc. 263:416. http://www.atnf.csiro.au/research/cmbr/cmbr_atca.html

[97] BAM: Balloon Anisotropy Measurement uses a differential Fourier transform spectrometer to measure the spectrum of the anisotropy between 90 and $300 \mathrm{GHz}$. Results in astro-ph/9609108. http://cmbr.physics.ubc.ca/ experimental.html

[98] BEAST BEAST: http://www.deepspace.ucsb.edu/research/Sphome.htm

[99] Bartol. A bolometer-based experiment designed to look at $2^{\circ}$ angular scales. Observed from the Canary Islands. Results in Piccirillo et al., astro-ph/9609186.

[100] BOOMERanG is a collaboration between the Caltech, Berkeley, Santa Barbara (Ruhl) and Rome groups. It will use bolometers to measure the anisotropy in the CMB between 90 and $410 \mathrm{GHz}$. BOOMERANG was designed for a circumpolar Antarctic flight. http://astro.caltech.edu/ lgg/boom/boom.html

[101] CAT: the Cambridge Anisotropy Telescope is an interferometer operating at $15 \mathrm{GHz}$ to image CMB anisotropies. Results reported in Scott et al., Astrophys. J. (1996) 461:L1. http://www.mrao.cam.ac.uk/telescopes/cat/index.html

[102] CBI: Cosmic Background Imager is an interferometer to map the microwave sky near $30 \mathrm{GHz}$. http://phobos.caltech.edu/ tjp/CBI/

[103] Cosmic Gene at RATAN 600 http://brown.nord.nw.ru/CG/CG.htm

[104] $C O B E$ is the COsmic Background Explorer. The three experiments aboard the satellite are the Differential Microwave Radiometers (30-90 GHz, DMR), the Far-InfraRed Absolute Spectrophotometer (60-630 GHz, FIRAS), and the Diffuse InfraRed Background Experiment (1.2-240 $\mu \mathrm{m}$, DIRBE) Eac experiment produced maps of the sky. http://www.gsfc.nasa.gov/astro/cobe/cobe_home.html and http://aether.lbl.gov/

[105] DASI: Degree Angular Scale Interferometer to map the CMB at $30 \mathrm{GHz}$ and is sensitive to larger angular scales than CBI. http://astro.uchicago.edu/dasi/

[106] FIRS, The Far InfraRed Survey, This is an experiment that started at MIT but has since moved to Princeton, University of Chicago and NASA/GSFC. It is a bolometer-based balloon-borne radiometer. It confirmed the initial COBE/DMR discovery. http://pupgg.princeton.edu/ cmb/firs.html

[107] HACME/SP, This uses HEMTs on the ACME telescope, observations were made from the South Pole. Recent results are reported in J. Gundersen et al., Astrophys. J. (1995) 443:L57. http://www.deepspace.ucsb.edu/research/Sphome.htm

[108] IAB, A bolometer-based experiment carried out at the Italian Antarctic Base. It observed the spectrum of the anisotropy between 90 and 300 GHz. Results are reported in L. Piccirillo and P. Calisse, Astrophys. J. (1993) 413:529.

[109] Bartol, This is a bolometer-based experiment designed to look at $2^{\circ}$ angular scales. It observed from the Canary Islands. Results are reported in Piccirillo et al., astro-ph/9609186.

[110] MAT: Mobile Anisotropy Telescope is similar to QMAP but operates from the ground in Chile. http://imogen.princeton.edu/ page/matdir/www/index.html

[111] MAX was a collaboration between UCSB and Berkeley. It is a balloon-borne bolometer-based radiometer spanning roughly between 90 and $420 \mathrm{GHz}$. Recent results are reported in Lim et al., Astrophys. J. (1996) 469:L69. It flew on the ACME telescope. http://cfpa.berkeley.edu/group/cmb/gen.html

[112] MAXIMA, MAX Imaging Array is a balloon-borne bolometer array. It is the next generation of MAX http://physics7.berkeley.edu/group/cmb/gen.html

[113] MSAM, There are a number of versions of MSAM. All use bolometers of various sorts and fly on balloons. The MSAM collaboration includes NASA/GSFC, Bartol Research Institute, Brown University, and the University of Chicago. http://topweb.gsfc.nasa.gov/

[114] OVRO, The Owen's Valley Radio Observatoty telescopes operate with various receivers between 15 and $30 \mathrm{GHz}$. The $40 \mathrm{~m}$ dish has a 2' beam, and the $5.5 \mathrm{~m}$ has a $7.3^{\prime}$ beam. The experiments are aimed primarily at small angular scales. http://www.cco.caltech.edu/ emleitch/ovro/ovro_cmb.html

[115] Planck http://astro.estec.esa.nl/Planck

[116] POLAR http://cmb.physics.wisc.edu/polar/

[117] Polatron: http://phobos.caltech.edu/ lgg/polatron/polatron.html 
[118] PYTHON. A multi-pixel bolometer- and HEMT-based experiment operated from the ground at the South Pole. The experiment has run in a number of configurations. Results reported in K. Coble et al., 1999, preprint astro-ph/9902195 http://cmbr.phys.cmu.edu/pyth.html

[119] QMAP is a balloon-borne combination of HEMTs and SIS detectors. The angular resolution is $1 / 5^{\circ}$. http://pupgg.princeton.edu/ cmb/qmap/qmap.html

[120] Ryle http://www.mrao.cam.ac.uk/ryle/index.html

[121] SASKATOON was a set of experiments based on HEMT amplifiers operating between 26 and $46 \mathrm{GHz}$. They were performed in Saskatoon, Saskatchewan CA. Three years of observations have gone into the final data set. http://pupgg.princeton.edu/ cmb/skintro/sask_intro.html

[122] SPort http://tonno.tesre.bo.cnr.it/ stefano/sp_draft.html

[123] SuZIE makes bolometer-based observations from the ground. Primarily aimed at measuring the SZ effect at high frequencies, it also gives information on the anisotropy at small scales. http://phobos.caltech.edu/ $\sim \operatorname{lgg} /$ suzie/suzie.html

[124] Tenerife, Ground-based differential radiometers with $10-33 \mathrm{GHz}$ receivers. The resolution is about $6^{\circ}$. The experiment observes from the Observatorio del Teide in Tenerife, Spain. It has operated for many years. http://clarin.ll.iac.es/

[125] TOPHAT is a collaboration between Bartol Research Institute, Brown University, DSRI, NASA/GSFC, and the University of Chicago. The group plans to observe with an extremely light-weight bolometer-based payload mounted on top of a scientific balloon that circumnavigates the Antarctic. For more information see http://topweb.gsfc.nasa.gov/

[126] Viper: http://cmbr.phys.cmu.edu/vip.html

[127] VLA, This is work done near $5 \mathrm{GHz}$, on arcminute and smaller angular scales. It uses the Very Large Array. Recent results are reported in Fomalont et al., Astrophys. J. (1993) 404:8-20. http://www.nrao.edu/vla/html/ VLAhome.shtml

[128] VSA: Very Small Array. This is a $30 \mathrm{GHz}$ interferometer; the next generation of CAT. http://www.mrao.com.ac.uk/ telescopes/cat/vsa.html

[129] White Dish, This experiment uses and on-axis Cassegrain telescope and a $90 \mathrm{GHz}$ single-mode bolometer. It observed at the South Pole and is sensitive to small angular scales. Results are reported in Tucker et al., Astrophys. J. (1993) 419:L45. http://www.cmbr.phys.cmu.edu/whitedish.html 